\title{
On hydromagnetic channel flow of an Oldroyd-B fluid induced by rectified sine pulses
}

\author{
A.K. GHOSH ${ }^{1}$ and PINTU SANA ${ }^{2}$ \\ ${ }^{1}$ Retd. Professor of Mathematics, Jadavpur University, Kolkata-70032, India \\ ${ }^{2}$ Teacher, Andrew's High (H.S) School, 33 Gariahat Road(S), Kolkata-700031, India \\ E-mail: akg10143@yahoo.co.in
}

\begin{abstract}
The unsteady unidirectional motion of an incompressible electrically conducting Oldroyd-B fluid in a channel bounded by two infinite rigid non-conducting parallel plates in presence of an external magnetic field acting in a direction normal to the plates has been discussed in this paper. The flow is supposed to generate impulsively from rest due to rectified sine pulses applied periodically on the upper plate with the lower plate held fixed. There is no external electric field imposed on the system and the magnetic Reynolds number is very small. Exact solution of the problem is obtained both by the methods of Fourier analysis and the Laplace transforms. The enquiries are made about the velocity field and the skin-friction on the walls. The influence of the magnetic field and the elasticity on the flow as well as on the skin-friction are examined quantitatively. Finally, it is shown that the expressions for the fluid velocity obtained by the method of Fourier analysis and by the method of Laplace transforms coincide to provide the same exact solution of the problem.
\end{abstract}

Mathematical subject classification: $76 \mathrm{~A} 10$.

Key words: hydromagnetic, pulsatile flow, Oldroyd-B fluid.

\section{Introduction}

It is well-known that the fluid flow generated by pulsatile motion of the boundary has important applications in aerodynamics, nuclear technology, astrophysics, geophysics, atmospheric science and cosmical gas dynamics. The investigation 
in this direction was presented by Chakraborty and Ray [1] who examined the magneto-hydrodynamic Couette flow between two parallel plates when one of the plates is set in motion by random pulses. Makar [2] presented the solution of magneto-hydrodynamic flow between two parallel plates when one of the plates is subjected to velocity tooth pulses and the induced magnetic field is neglected. Bestman and Njoku [3] constructed solution of hydrodynamic channel flow of an incompressible, electrically conducting viscous fluid induced by tooth pulses including the effect of induced magnetic field, ignored by the author [2], and using the methodology of Fourier analysis instead of applying the commonly used technique of Laplace transforms which involve complicated inversions. Ghosh and Debnath [4] considered the hydromagnetic channel flow of a two-phase fluidparticle system induced by tooth pulses and obtained solution using the method of Laplace transforms. Datta and Dalal $[5,6]$ discussed the pulsatile flow and heat transfer of a dusty fluid in a channel and in an annular pipe employing the method of perturbation. On the other hand, Hayat et al. [7] have studied some simple flows of an Oldroyd-B fluid using the method of Fourier transforms. Asghar et al. [8] also utilized the same methodology as that of authors [7] to solve the problem concerning Hall effect on unsteady hydromagnetic flows of an Oldroyd-B fluid while Hayat et al. [9] constructed the solution of hydromagnetic Couette flow of an Oldroyd-B fluid in a rotating system following the method of perturbation. In the present paper, the problem as that of author [2] has been studied in the case of an Oldroyd-B fluid which takes into account both the elastic and memory effects exhibited by most polymeric and biological liquids when the upper plate is set in motion by rectified sine pulses instead of velocity tooth pulses as considered by earlier authors [2, 3, 4]. It appears that, besides various applications mentioned above, the present investigation is particularly useful in powder and polymer technology and in detecting the effect of magnetic field on electrically conducting physiological fluid flow systems [10].

The problem is concerned with the unsteady hydromagnetic flow of an incompressible, electrically conducting Oldryod-B fluid in a channel bounded by two infinite rigid non-conducting parallel plates. The motion is suppose to start from rest in the fluid due to rectified sine pulses applied periodically on the upper plate with the lower plate held fixed. Exact expression for the fluid velocity is obtained 
using the methods of Fourier analysis and Laplace transforms separately. The results for the skin-friction on the walls are also obtained in both the cases. It is shown that both the methods give the same exact solution of the problem. The effects of the magnetic field and the elasticity on the developing and the retarding flows and also on the skin-friction at the plates are discussed quantitatively. It is observed that the viscoelastic flows grow and decay less faster than the ordinary viscous fluids. The magnetic field has a damping effect on such flows. Both the elastic and magnetic effects reduce with the increase of time period of oscillations of the plate irrespective of the nature of the flow so that a classical hydrodynamic situation arises when the frequency of oscillations is very small.

\section{Basic equations}

The constitutive equations for an Oldroyd-B fluid [7-9] are

$$
\begin{aligned}
\mathbf{T} & =-p \mathbf{I}+\mathbf{S} \\
\mathbf{S}+\lambda_{1} \frac{D \mathbf{S}}{D t} & =\mu\left[1+\lambda_{2} \frac{D}{D t}\right] \mathbf{A}_{\mathbf{1}}
\end{aligned}
$$

where $\mathbf{T}=$ Cauchy stress tensor, $p=$ fluid pressure, $\mathbf{I}=$ identity tensor, $\mathbf{S}=$ extra stress tensor, $\mu, \lambda_{1}, \lambda_{2}=$ viscosity coefficient, relaxation time, retardation time (assumed constants).

The tensor $\mathbf{A}_{1}$ is defined as

$$
\mathbf{A}_{\mathbf{1}}=\nabla \mathbf{V}+(\nabla \mathbf{V})^{T}
$$

In a cartesian system, $\frac{D}{D t}$ (upper convected time derivative) operating on any tensor $\mathbf{B}_{\mathbf{1}}$ is

$$
\frac{D \mathbf{B}_{\mathbf{1}}}{D t}=\frac{\partial \mathbf{B}_{\mathbf{1}}}{\partial t}+(\mathbf{V} \cdot \nabla) \mathbf{B}_{\mathbf{1}}-(\nabla \mathbf{V}) \mathbf{B}_{\mathbf{1}}-\mathbf{B}_{\mathbf{1}}(\nabla \mathbf{V})^{T} .
$$

It is to be mentioned here that this model includes the viscous fluid as a particular case for $\lambda_{1}=\lambda_{2}$, the Maxwell fluid when $\lambda_{2}=0$ and an Oldroyd-B fluid when $0<\lambda_{2}<\lambda_{1}<1$. The stress equations of motion for an incompressible electrically conducting Oldroyd-B fluid in presence of an external magnetic fluid are

$$
\nabla \cdot \mathbf{V}=0
$$




$$
\begin{gathered}
\rho\left[\frac{\partial \mathbf{V}}{\partial t}+(\mathbf{V} \cdot \nabla) \mathbf{V}\right]=\nabla \cdot \mathbf{T}+\mathbf{J} \times \mathbf{B}, \\
\nabla \cdot \mathbf{B}=0, \quad \nabla \times \mathbf{B}=\mu_{0} \mathbf{J}, \\
\nabla \times \mathbf{E}=-\frac{\partial \mathbf{B}}{\partial t}, \quad \mathbf{J}=\sigma[\mathbf{E}+\mathbf{V} \times \mathbf{B}]
\end{gathered}
$$

where $\mathbf{V}=(\mathbf{u}, \mathbf{v}, \mathbf{w})=$ fluid velocity, $\rho=$ fluid density, $\mathbf{J}=$ current density, $\mathbf{B}=$ magnetic flux density, $\mathbf{E}=$ electric field, $\mu_{0}=$ magnetic permeability (assumed constant), $\sigma=$ electrical conductivity (assumed finite).

\section{Formulation of the problem}

In this problem, we consider the motion of an incompressible electrically conducting Oldroyd-B fluid between two infinite rigid non-conducting parallel plates separated by a distance $h$. The $x$-axis is taken in the direction of flow with origin at the lower plate and $y$-axis perpendicular to the plates. The initial motion is generated in the fluid due to rectified sine pulses applied on the upper plate. The lower plate is held fixed. A uniform magnetic field of strength $B_{0}$ is acting parallel to $y$-axis. We assume that no external electric field is acting on the fluid and the magnetic Reynolds number is very small. This implies that the current is mainly due to induced electric field and the applied magnetic field remains essentially unaltered by the electric current flowing in the fluid. We also assume that the induced magnetic field produced by the motion of the fluid is negligible compared to the applied magnetic field so that the Lorentz force term in (2.6) becomes $-\sigma B_{0}^{2} \mathbf{V}$.

Since the motion is a plain one and the plates are infinitely long, we assume that all the physical variables are independent of $x$ and $z$. Then from the equation of continuity (2.5) and from the physical condition of the problem, we take

$$
\mathbf{V}=\{u(y, t), 0,0\} \quad \text { and } \quad \mathbf{S}=\mathbf{S}(y, t) .
$$

The equations of motion in (2.6) then reduces to

$$
\begin{gathered}
\rho \frac{\partial u}{\partial t}=-\frac{\partial p}{\partial x}+\frac{\partial S_{x y}}{\partial y}-\sigma B_{0}^{2} u, \\
\frac{\partial p}{\partial y}=\frac{\partial S_{y y}}{\partial y},
\end{gathered}
$$




$$
\frac{\partial p}{\partial z}=0
$$

It follows from (2.2) and (3.1 ab) that

$$
\begin{gathered}
S_{x x}+\lambda_{1}\left[\frac{\partial S_{x x}}{\partial t}-2 S_{x y} \frac{\partial u}{\partial y}\right]=-2 \mu \lambda_{2}\left(\frac{\partial u}{\partial y}\right)^{2}, \\
S_{x y}+\lambda_{1}\left[\frac{\partial S_{x y}}{\partial t}-S_{y y} \frac{\partial u}{\partial y}\right]=\mu\left(\frac{\partial u}{\partial y}\right)+\lambda_{2} \mu\left(\frac{\partial^{2} u}{\partial y \partial t}\right), \\
S_{y y}+\lambda_{1} \frac{\partial S_{y y}}{\partial t}=0 .
\end{gathered}
$$

The equation (3.7) gives

$$
S_{y y}=A(y) e^{-t / \lambda_{1}}
$$

where $A(y)$ is an arbitrary function of $y$. But $S_{y y}$ is known to be zero for $t<0$. This implies that $A(y)$ must be zero. Hence $S_{y y}$ is zero always. Consequently, from (3.2) and (3.6) and in absence of pressure gradient along $x$ direction, we get

$$
\left(1+\lambda_{1} \frac{\partial}{\partial t}\right) \frac{\partial u}{\partial t}=v\left(1+\lambda_{2} \frac{\partial}{\partial t}\right) \frac{\partial^{2} u}{\partial y^{2}}-\frac{\sigma B_{0}^{2}}{\rho}\left(1+\lambda_{1} \frac{\partial}{\partial t}\right) u
$$

which on introducing the dimensionless quantities given by

$$
\begin{gathered}
\bar{u}=\frac{u}{U_{0}}, \bar{y}=\frac{y}{\sqrt{v \lambda_{1}}}, \quad \bar{t}=\frac{t}{\lambda_{1}}, d=\frac{h}{\sqrt{v \lambda_{1}}}, \\
k=\frac{\lambda_{2}}{\lambda_{1}}(\leq 1) \quad \text { and } \quad M^{2}=\frac{\sigma B_{0}^{2} \lambda_{1}}{\rho}
\end{gathered}
$$

and on dropping the bars, we get

$$
\left(1+\frac{\partial}{\partial t}\right) \frac{\partial u}{\partial t}=\left(1+k \frac{\partial}{\partial t}\right) \frac{\partial^{2} u}{\partial y^{2}}-M^{2}\left(1+\frac{\partial}{\partial t}\right) u .
$$

The problem now reduces to solving (3.10) subject to boundary and initial conditions:

$$
u(0, t)=0, u(d, t)=f(t) \text { for all } t>0
$$

and

$$
u(y, 0)=0, u_{t}(y, 0)=0 \text { for } 0 \leq y \leq d
$$

where $f(t)$ representing the rectified sine pulses, as shown in Figure 1, is an even periodic function with period $2 \mathrm{~T}$. 


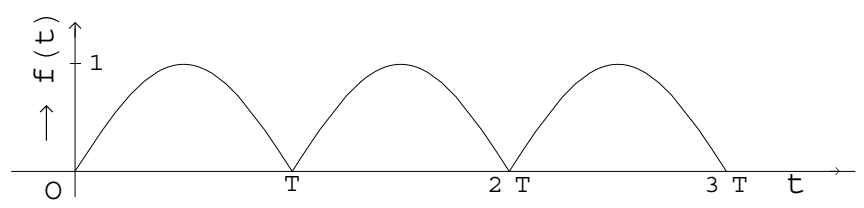

Figure 1 - Rectified sine pulses.

\section{Solution of the problem}

\section{Method of Fourier analysis}

According to the nature of $f(t)$ mentioned above, the mathematical form of $u(d, t)$ may be written as

$$
u(d, t)=\sin \frac{\pi t}{T} H(t)+2 \sum_{m=1}^{\infty} \sin \frac{\pi(t-m T)}{T} H(t-m T)
$$

where $H(t-T)=0, t<T$ and $H(t-T)=1, t \geq T$.

Using half-range Fourier series, the condition (4.1) can also be expressed in the form

$$
u(d, t)=\frac{2}{\pi}-\frac{4}{\pi} \sum_{m=1}^{\infty} \frac{1}{(2 m)^{2}-1} \cos \left\{\frac{2 m \pi t}{T}\right\} .
$$

By virtue of (4.2) we assume the solution of (3.10) as

$$
\begin{aligned}
u(y, t)= & u_{s}(y)+\frac{1}{2} \sum_{m=1}^{\infty}\left[u_{2 m}(y) e^{i \frac{2 m \pi t}{T}}+\bar{u}_{2 m}(y) e^{-i \frac{2 m \pi t}{T}}\right] \\
& +\sum_{n=1}^{\infty} W_{n}(t) \sin \left(\frac{n \pi y}{d}\right)
\end{aligned}
$$

where $\bar{u}$ is the conjugate of $u$. The first two terms in (4.3) are chosen so as to satisfy (4.2) while the last term accommodates the initial condition.

Substituting (4.3) in (3.10) and then using (4.2), we have the following equations with appropriate conditions as

$$
\frac{d^{2} u_{s}}{d y^{2}}-M^{2} u_{s}=0
$$

with $u_{s}=0$ on $y=0, u_{s}=\frac{2}{\pi}$ on $y=d$,

$$
\frac{d^{2} u_{2 m}}{d y^{2}}-L_{m}^{2} u_{2 m}=0
$$


with $u_{2 m}=0$ on $y=0, u_{2 m}=-\frac{4}{\pi} \frac{1}{(2 m)^{2}-1}$ on $y=d$ and

$$
\frac{d^{2} W_{n}}{d t^{2}}+\left(1+M^{2}+\frac{n^{2} \pi^{2} k}{d^{2}}\right) \frac{d W_{n}}{d t}+\left(M^{2}+\frac{n^{2} \pi^{2}}{d^{2}}\right) W_{n}=0
$$

with $W_{n}=W_{n}(0), W_{n}^{\prime}=W_{n}^{\prime}(0)$ at $t=0$ where $W_{n}(0)$ and $W_{n}^{\prime}(0)$ are to be determined.

In the above,

$$
L_{m}^{2}=\frac{\left(M^{2}+i \beta_{m}\right)\left(1+i \beta_{m}\right)}{1+i \beta_{m} k} \text { and } \beta_{m}=\frac{2 m \pi}{T} .
$$

The solutions of equations (4.4)-(4.6) are

$$
\begin{gathered}
u_{s}(y)=\frac{2}{\pi} \frac{\sinh M y}{\sinh M d} \\
u_{2 m}(y)=-\frac{4}{\pi} \frac{1}{(2 m)^{2}-1} \frac{\sinh L_{m} y}{\sinh L_{m} d} \\
W_{n}(t)=W_{n}^{\prime}(0) \frac{e^{m_{1} t}-e^{m_{2} t}}{m_{1}-m_{2}}+W_{n}(0) \frac{m_{1} e^{m_{2} t}-m_{2} e^{m_{1} t}}{m_{1}-m_{2}}
\end{gathered}
$$

where

$$
\begin{gathered}
2 m_{1}, 2 m_{2}=-\left[\left(1+M^{2}+\frac{n^{2} \pi^{2} k}{d^{2}}\right)\right. \\
\left.\mp\left\{\left(1+M^{2}+\frac{n^{2} \pi^{2} k}{d^{2}}\right)^{2}-4\left(M^{2}+\frac{n^{2} \pi^{2}}{d^{2}}\right)\right\}^{1 / 2}\right] .
\end{gathered}
$$

The initial conditions in (3.12) provide

$$
\begin{gathered}
W_{n}(0)= \\
4 n(-1)^{n}\left[\frac{1}{n^{2} \pi^{2}+M^{2} d^{2}}-2 \operatorname{Re} \sum_{m=1}^{\infty} \frac{1}{\left\{(2 m)^{2}-1\right\}\left(n^{2} \pi^{2}+L_{m}^{2} d^{2}\right)}\right], \\
W_{n}^{\prime}(0)=8 n(-1)^{n} \operatorname{Im} \sum_{m=1}^{\infty} \frac{\beta_{m}}{\left\{(2 m)^{2}-1\right\}\left(n^{2} \pi^{2}+L_{m}^{2} d^{2}\right)}
\end{gathered}
$$

where Re and Im stand respectively for the real and the imaginary parts of the above expressions. 
The $m$-series in (4.11) and (4.12) are of orders $\beta_{m}^{-3}$ and $\beta_{m}^{-2}$ when $m \longrightarrow \infty$. The $n$-series is also convergent since $m_{1}, m_{2}$ are of order $-N_{1}^{2}$ and $m_{1}-m_{2}$ has the order $N_{1}^{2}$ as $n \longrightarrow \infty$ where $N_{1}^{2}=M^{2}+\frac{n^{2} \pi^{2} k}{d^{2}}$.

Finally, the fluid velocity takes the form

$$
\begin{gathered}
u(y, t)=\frac{2 \sinh M y}{\pi \sinh M d}-\frac{4}{\pi} \operatorname{Re} \sum_{m=1}^{\infty} \frac{e^{i \beta_{m} t}}{(2 m)^{2}-1} \frac{\sinh L_{m} y}{\sinh L_{m} d} \\
+\sum_{n=1}^{\infty}\left\{W_{n}^{\prime}(0) \frac{e^{m_{1} t}-e^{m_{2} t}}{m_{1}-m_{2}}+W_{n}(0) \frac{m_{1} e^{m_{2} t}-m_{2} e^{m_{1} t}}{m_{1}-m_{2}}\right\} \sin \frac{n \pi y}{d}
\end{gathered}
$$

which in the limit $t \longrightarrow \infty$ provides the steady velocity field

$$
u(y, t)=\frac{2 \sinh M y}{\pi \sinh M d}-\frac{4}{\pi} \operatorname{Re} \sum_{m=1}^{\infty} \frac{e^{i \beta_{m} t}}{(2 m)^{2}-1} \frac{\sinh L_{m} y}{\sinh L_{m} d}
$$

where the harmonic part contains the effect of elasticity in presence of pulsation.

On the other hand, the solution corresponding to classical viscous fluid can be obtained from (4.13) in the limit $k \longrightarrow 1$. This solution is given by

$$
\begin{gathered}
u(y, t)=\frac{2 \sinh M y}{\pi \sinh M d}-\frac{4}{\pi} \operatorname{Re} \sum_{m=1}^{\infty} \frac{e^{i \beta_{m} t}}{(2 m)^{2}-1} \frac{\sinh L_{m}^{*} y}{\sinh L_{m}^{*} d} \\
+\sum_{n=1}^{\infty} 4 n(-1)^{n}\left[\frac{1}{M^{2} d^{2}+n^{2} \pi^{2}}\right. \\
\left.-2 \sum_{m=1}^{\infty} \frac{1}{(2 m)^{2}-1} \frac{M^{2} d^{2}+n^{2} \pi^{2}}{\left(M^{2} d^{2}+n^{2} \pi^{2}\right)^{2}+\beta_{m}^{2} d^{4}}\right] e^{-\left(M^{2}+n^{2} \pi^{2} / d^{2}\right) t} \sin \frac{n \pi y}{d}
\end{gathered}
$$

where $L_{m}^{*}=\sqrt{M^{2}+i \beta_{m}}$.

The result (4.15) is identical to that of Bestman and Njoku [3].

In particular, when $T \longrightarrow 0$ the result (4.13) reduces to

$$
u(y, t)=\frac{2 \sinh M y}{\pi \sinh M d}+4 \sum_{n=1}^{\infty} \frac{n(-1)^{n}}{M^{2} d^{2}+n^{2} \pi^{2}} \frac{m_{1} e^{m_{2} t}-m_{2} e^{m_{1} t}}{m_{1}-m_{2}} \sin \frac{n \pi y}{d}
$$

which describes the hydromagnetic channel flow of an Oldroyd-B fluid generated by impulsive motion of the upper plate with a constant velocity. The result (4.16) in dimensional form, in the limit $M \longrightarrow 0$, coincides exactly with that of 
authors [7] while the result (4.15) in the limit $T \longrightarrow 0$ agrees completely with that of Soundalgekar [11].

The skin-friction on the plates $y=0$ and $y=d$ are given by

$$
\begin{aligned}
\tau_{0}= & \frac{2 M\left(1-e^{-t}\right)}{\pi \sinh M d}-\frac{4}{\pi} \operatorname{Re} \sum_{m=1}^{\infty} \frac{L_{m}}{(2 m)^{2}-1} \frac{1+i \beta_{m} k}{1+i \beta_{m}} \frac{e^{i \beta_{m} t}-e^{-t}}{\sinh L_{m} d} \\
& +\sum_{n=1}^{\infty} \frac{n \pi}{d}\left\{\frac{W_{n}^{\prime}(0)}{m_{1}-m_{2}}\left[\frac{1+k m_{1}}{1+m_{1}}\left(e^{m_{1} t}-e^{-t}\right)-\frac{1+k m_{2}}{1+m_{2}}\left(e^{m_{2} t}-e^{-t}\right)\right]\right. \\
& \left.+\frac{W_{n}(0)}{m_{1}-m_{2}}\left[\frac{\left(1+k m_{2}\right) m_{1}}{1+m_{2}}\left(e^{m_{2} t}-e^{-t}\right)-\frac{\left(1+k m_{1}\right) m_{2}}{1+m_{1}}\left(e^{m_{1} t}-e^{-t}\right)\right]\right\}, \\
\tau_{d}= & \frac{2 M \cosh M d}{\pi \sinh M d}\left(1-e^{-t}\right)-\frac{4}{\pi} \operatorname{Re} \sum_{m=1}^{\infty} \frac{L_{m}}{(2 m)^{2}-1} \\
& \times \frac{1+i \beta_{m} k}{1+i \beta_{m}}\left(e^{i \beta_{m} t}-e^{-t}\right) \frac{\cosh L_{m} d}{\sinh L_{m} d} \\
& +\sum_{n=1}^{\infty} \frac{n \pi}{d}(-1)^{n}\left\{\frac{W_{n}^{\prime}(0)}{m_{1}-m_{2}}\left[\frac{1+k m_{1}}{1+m_{1}}\left(e^{m_{1} t}-e^{-t}\right)-\frac{1+k m_{2}}{1+m_{2}}\left(e^{m_{2} t}-e^{-t}\right)\right]\right. \\
& \left.+\frac{W_{n}(0)}{m_{1}-m_{2}}\left[\frac{\left(1+k m_{2}\right) m_{1}}{1+m_{2}}\left(e^{m_{2} t}-e^{-t}\right)-\frac{\left(1+k m_{1}\right) m_{2}}{1+m_{1}}\left(e^{m_{1} t}-e^{-t}\right)\right]\right\} .
\end{aligned}
$$

Above results in the limit $k \longrightarrow 1$ (viscous fluid) reduces to

$$
\begin{aligned}
\tau_{0}= & \frac{2 M}{\pi \sinh M d}-\frac{4}{\pi} \operatorname{Re} \sum_{m=1}^{\infty} \frac{e^{i \beta_{m} t}}{(2 m)^{2}-1} \frac{L_{m}^{*}}{\sinh L_{m}^{*} d} \\
& +\sum_{n=1}^{\infty} \frac{4 \pi n^{2}(-1)^{n}}{d} \frac{e^{-\left(M^{2}+\frac{n^{2} \pi^{2}}{d^{2}}\right) t}}{M^{2} d^{2}+n^{2} \pi^{2}}
\end{aligned}
$$

and

$$
\begin{aligned}
\tau_{d}= & \frac{2 M \cosh M d}{\pi \sinh M d}-\frac{4}{\pi} \operatorname{Re} \sum_{m=1}^{\infty} \frac{e^{i \beta_{m} t}}{(2 m)^{2}-1} \frac{L_{m}^{*} \cosh L_{m}^{*} d}{\sinh L_{m}^{*} d} \\
& +\sum_{n=1}^{\infty} \frac{4 \pi n^{2}}{d} \frac{e^{-\left(M^{2}+\frac{n^{2} \pi^{2}}{d^{2}}\right) t}}{M^{2} d^{2}+n^{2} \pi^{2}} .
\end{aligned}
$$


However, when $T \longrightarrow 0,(4.19)$ and (4.20) provide the classical hydromagnetic solutions given by

$$
\begin{aligned}
\tau_{0} & =\frac{2 M}{\pi \sinh M d}+\frac{4 \pi}{d} \sum_{n=1}^{\infty} \frac{n^{2}(-1)^{n}}{M^{2} d^{2}+n^{2} \pi^{2}} e^{-\left(M^{2}+\frac{n^{2} \pi^{2}}{d^{2}}\right) t}, \\
\tau_{d} & =\frac{2 M \cosh M d}{\pi \sinh M d}+\frac{4 \pi}{d} \sum_{n=1}^{\infty} \frac{n^{2}}{M^{2} d^{2}+n^{2} \pi^{2}} e^{-\left(M^{2}+\frac{n^{2} \pi^{2}}{d^{2}}\right) t} .
\end{aligned}
$$

\section{Method of Laplace transforms}

The problem, when solved by the method of Laplace transform technique, reduces to solving the transformed equation

$$
\frac{d^{2} \bar{u}}{d y^{2}}-\frac{(1+s)\left(s+M^{2}\right)}{1+k s} \bar{u}=0
$$

subject to the conditions

$$
\bar{u}=0 \text { at } y=0, \quad \bar{u}=\frac{\pi T}{T^{2} s^{2}+\pi^{2}} \operatorname{coth}\left(\frac{s T}{2}\right) \text { at } y=d .
$$

The transformed solution for the fluid velocity $\bar{u}(y, s)$ becomes

$$
\bar{u}(y, s)=\frac{\pi T}{T^{2} s^{2}+\pi^{2}} \operatorname{coth}\left(\frac{s T}{2}\right) \frac{\sinh L y}{\sinh L d}
$$

where $L^{2}=\frac{(1+s)\left(s+M^{2}\right)}{1+k s}$.

The inversion of (4.25) gives

$$
u(y, t)=\frac{\pi T}{2 \pi i} \int_{\gamma-i \infty}^{\gamma+i \infty} \frac{\exp (s t) \operatorname{coth}(s T / 2)}{T^{2} s^{2}+\pi^{2}} \frac{\sinh L y}{\sinh L d} d s .
$$

The integrand has a pole at $s=0$, a series of simple poles at $s= \pm i \beta_{m}$, $m=0,1,2, \ldots$, and simple poles at $s_{1}, s_{2}$ which are roots of $\sinh L d=0$.

Following Carslaw and Jaeger [12], the expression for the fluid velocity $u(y, t)$ takes the form

$$
\begin{aligned}
u(y, t)= & \frac{2 \sinh M y}{\pi \sinh M d}-\frac{4}{\pi} \operatorname{Re} \sum_{m=1}^{\infty} \frac{e^{i \beta_{m} t}}{(2 m)^{2}-1} \frac{\sinh L_{m} y}{\sinh L_{m} d} \\
& -\frac{2 \pi^{2}}{T d^{2}} \sum_{n=1}^{\infty} n(-1)^{n} G \sin \frac{n \pi y}{d}
\end{aligned}
$$


where

$$
\begin{gathered}
\beta_{m}=\frac{2 m \pi}{T}, \quad L_{m}=\left[\frac{\left(1+i \beta_{m}\right)\left(M^{2}+i \beta_{m}\right)}{1+i \beta_{m} k}\right]^{1 / 2}, \\
G=G_{1}+G_{2}, \quad G_{j}=\frac{e^{s_{j} t} \operatorname{coth} \frac{s_{j} T}{2}}{\left(s_{j}^{2}+\frac{\pi^{2}}{T^{2}}\right)\left\{a_{1}+\frac{b_{1}}{\left(1+k s_{j}\right)^{2}}\right\}}, j=1,2, \\
a_{1}=\frac{1}{k}, \quad b_{1}=\left(M^{2}-\frac{1}{k}\right)(1-k), \\
-\left[\left(1+M^{2}+\frac{n^{2} \pi^{2} k}{d^{2}}\right) \mp \sqrt{\left(1+M^{2}+\frac{n^{2} \pi^{2} k}{d^{2}}\right)^{2}-4\left(M^{2}+\frac{n^{2} \pi^{2}}{d^{2}}\right)}\right] .
\end{gathered}
$$

The result (4.27), in the limit $k \longrightarrow 1$, is in excellent agreement with those of author [2] and authors [4].

The corresponding expressions for skin-friction on the plates are

$$
\begin{aligned}
\tau_{0}= & \frac{2 M\left(1-e^{-t}\right)}{\pi \sinh M d} \\
& -\frac{4}{\pi} \operatorname{Re} \sum_{m=1}^{\infty}\left[\frac{L_{m}}{(2 m)^{2}-1} \frac{1+i \beta_{m} k}{1+i \beta_{m}} \frac{e^{i \beta_{m} t}-e^{-t}}{\sinh L_{m} d}\right] \\
& -\frac{2 \pi^{3}}{T d^{3}} \sum_{n=1}^{\infty} n^{2}(-1)^{n} H \\
\tau_{d}= & \frac{2 M \cosh M d}{\pi \sinh M d}\left(1-e^{-t}\right) \\
& -\frac{4}{\pi} \operatorname{Re} \sum_{m=1}^{\infty}\left[\frac{L_{m}}{(2 m)^{2}-1} \frac{1+i \beta_{m} k}{1+i \beta_{m}}\left(e^{i \beta_{m} t}-e^{-t}\right) \frac{\cosh L_{m} d}{\sinh L_{m} d}\right] \\
& -\frac{2 \pi^{3}}{T d^{3}} \sum_{n=1}^{\infty} n^{2} H
\end{aligned}
$$

where

$$
H=H_{1}+H_{2}, \quad H_{j}=\frac{1+k s_{j}}{1+s_{j}} \frac{\left(e^{s_{j} t} e^{-t}\right) \operatorname{coth} \frac{s_{j} T}{2}}{\left(s_{j}^{2}+\frac{\pi^{2}}{T^{2}}\right)\left\{a_{1}+\frac{b_{1}}{\left(1+k s_{j}\right)^{2}}\right\}}, j=1,2 .
$$


The result (4.13) obtained by the method of Fourier analysis when compared with (4.27) obtained by the method of Laplace transforms reveals that the two results are exactly identical in respect of their steady and harmonic parts but the transient parts of them are of different forms. In order to show that these two results for the fluid velocity represent the same exact solution of the problem we incorporate the analysis given in the appendix.

\section{Numerical results}

The nature of the pulses applied on the upper plate, as shown in Figure 1, produces the developing (increasing) and the retarding (decreasing) flows during the first and the next half of each pulse respectively. Accordingly, the effect of the elasticity $(k)$ on the fluid velocity given by the equation (4.13), for both the developing and retarding flows, are shown in the Figures 2(a), 2(b) and 2(c) when $M=1.0$ and $T=0.5,1.0,2.0$. In the Figure 2(a) the velocity profiles for $t=0.125$ and $t=0.25$ represent the developing flows and those for $t=0.375$ and $t=0.5$ stand for the retarding flows. Further enquiry shows that the fluid velocity increases with the increase of the elastic parameter $k$ when the flow is developing (Table 1) and decreases with the increase of $k$ when the flow is retarding (Table 2). It is to be noted here that the fluid will be highly viscoelastic for smaller and smaller values of $k$. The results corresponding to the value of $k=1$ describe the Newtonian flow which is free from elastic effect. Consequently, it appears from Figures $2(a, b, c)$ that the viscoelastic fluids neither grow nor decay as quickly as Newtonian viscous fluids due to the restraining effect played by the elasticity of the fluid. Moreover, in the retarding motion, increasing effect of the elastic parameter $k$ prevails on the flow for some time until the diminishing effect of $k$ becomes dominant to nullify it completely (see Figure 2(a) at $t=0.375$, Table 2). This is expected. The Figures 2(b) and 2(c), represent the effects of elasticity $(k)$ on the velocity profiles with increasing values of time period $T$. On the other hand, the magnetic field $(M)$ has damping effect on the flow irrespective of its nature. This phenomena is shown in Figures 3(a), 3(b) and 3(c). Figure 4 provides a comparison how the increasing and decreasing effects produced by $k$ respectively on the developing and retarding flows are reduced with the increase of time period 


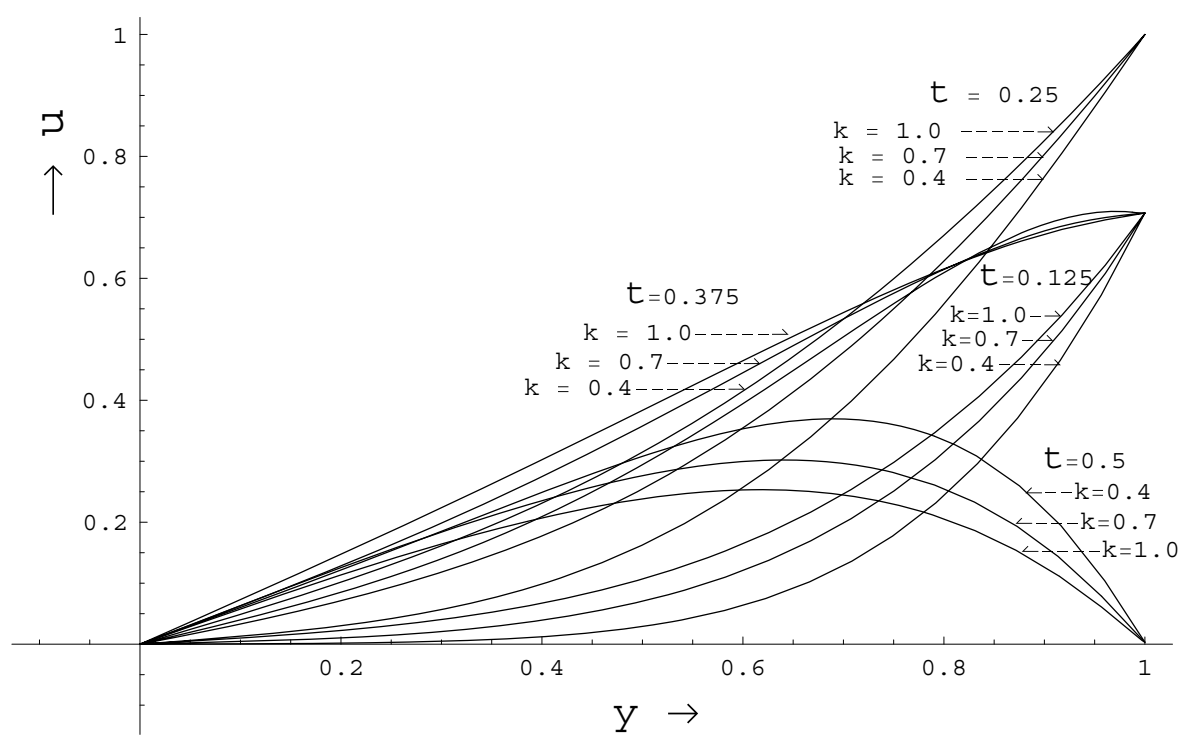

Figure 2(a) - Effect of elasticity $(k)$ on the fluid velocity when $T=0.5$ and $M=1.0$.

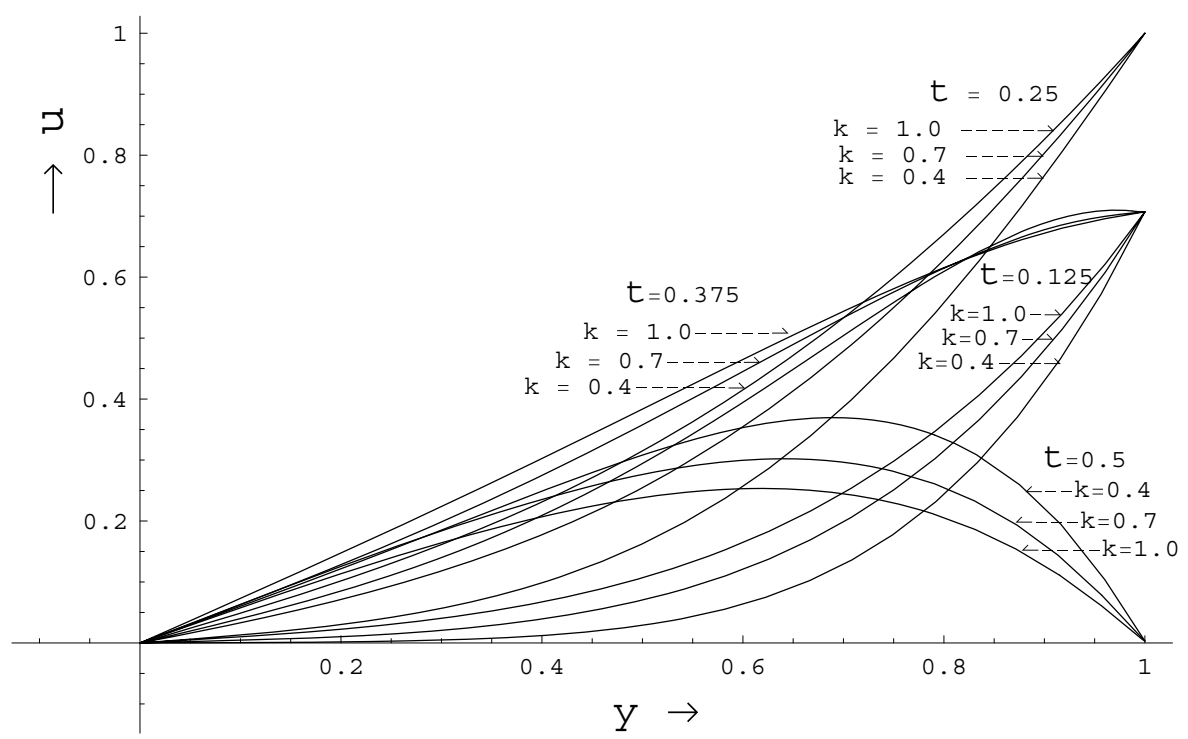

Figure 2(b) - Effect of elasticity $(k)$ on the fluid velocity when $T=1.0$ and $M=1.0$. 


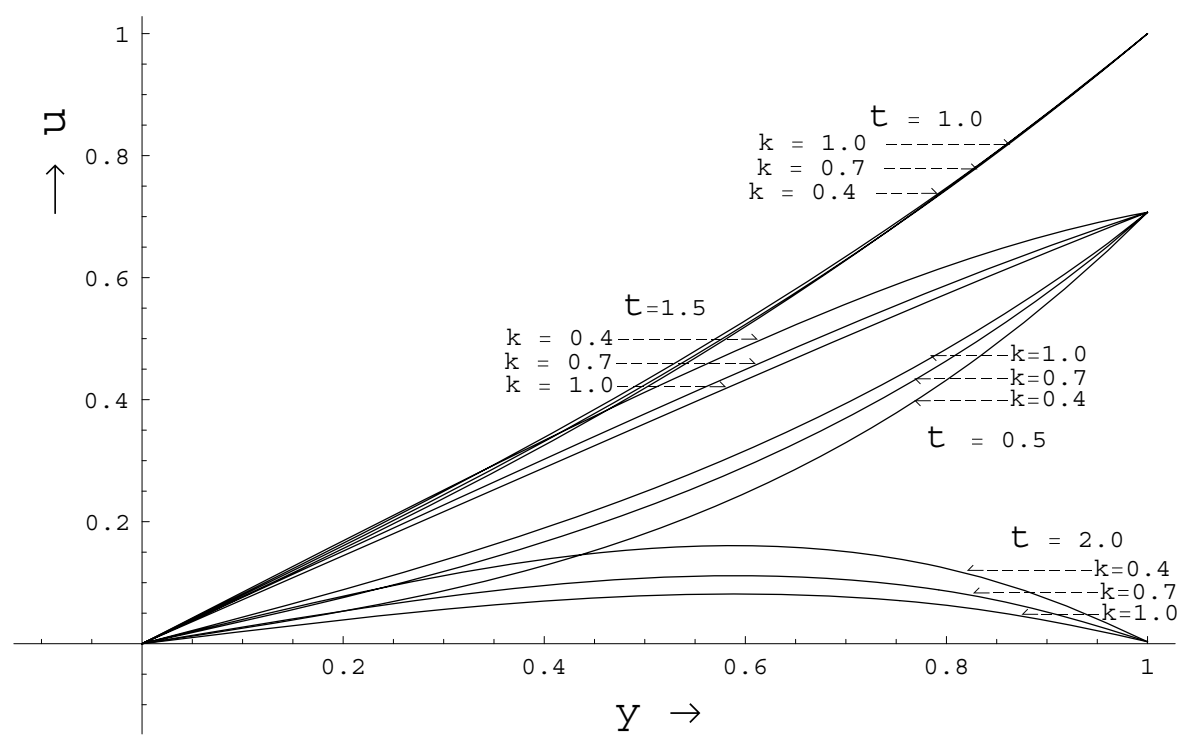

Figure 2(c) - Effect of elasticity $(k)$ on the fluid velocity when $T=2.0$ and $M=1.0$.

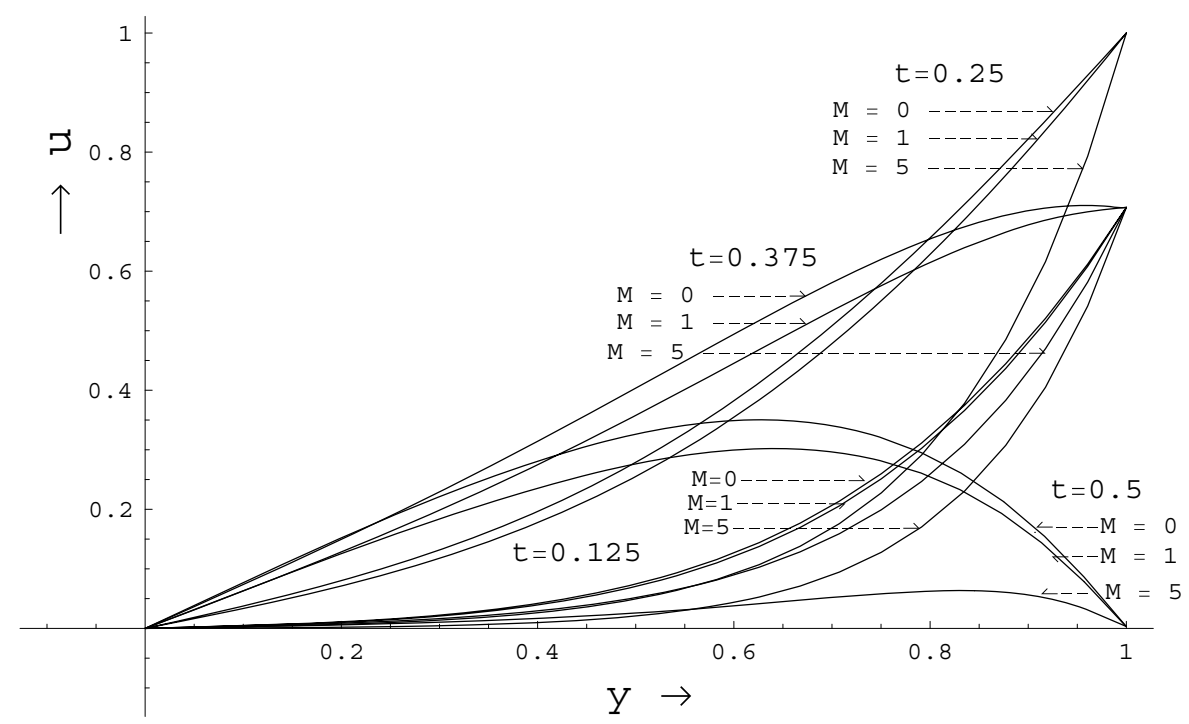

Figure 3(a) - Effect of the magnetic field $(M)$ on the fluid velocity when $T=0.5$ and $k=0.7$. 


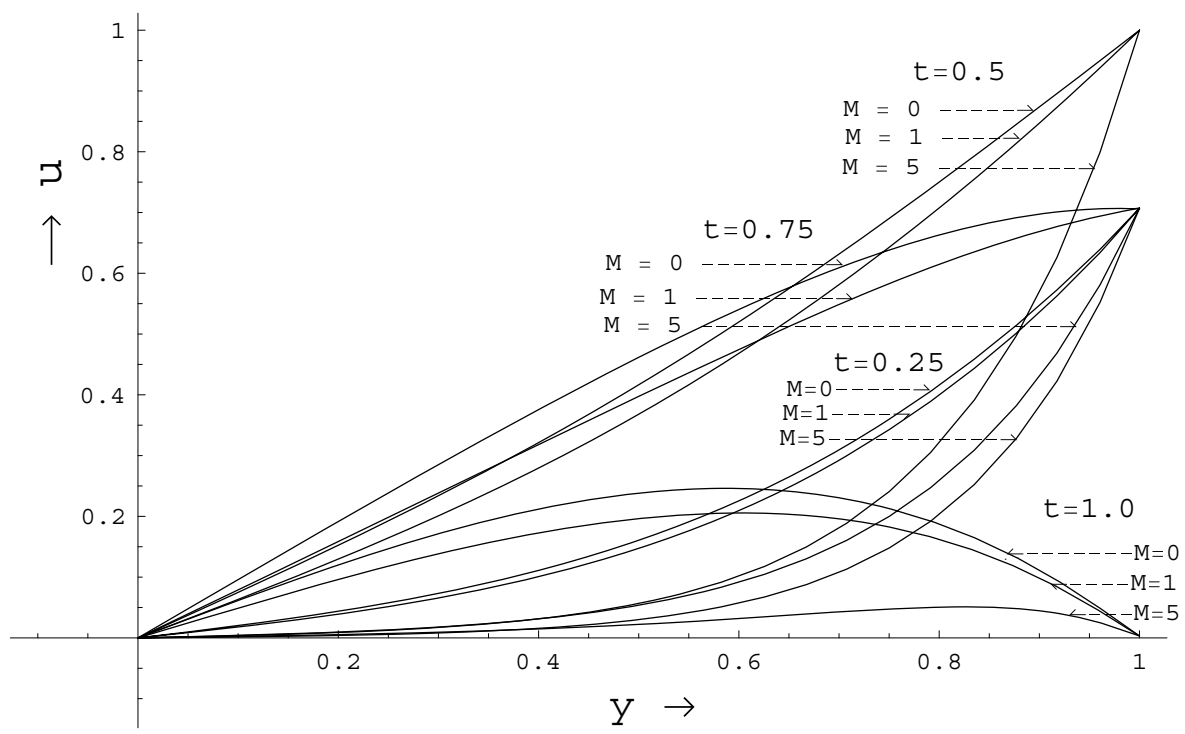

Figure 3(b) - Effect of the magnetic field $(M)$ on the fluid velocity when $T=1.0$ and $k=0.7$.

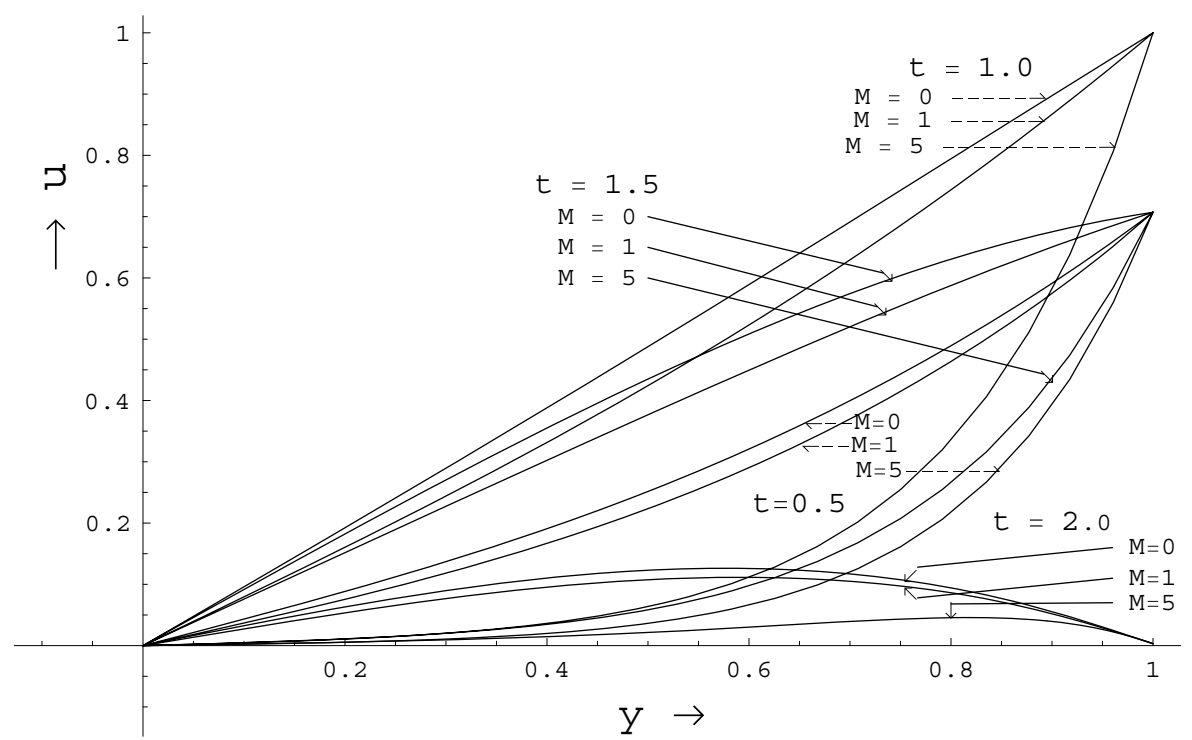

Figure 3(c) - Effect of the magnetic field $(M)$ on the fluid velocity when $T=2.0$ and $k=0.7$. 


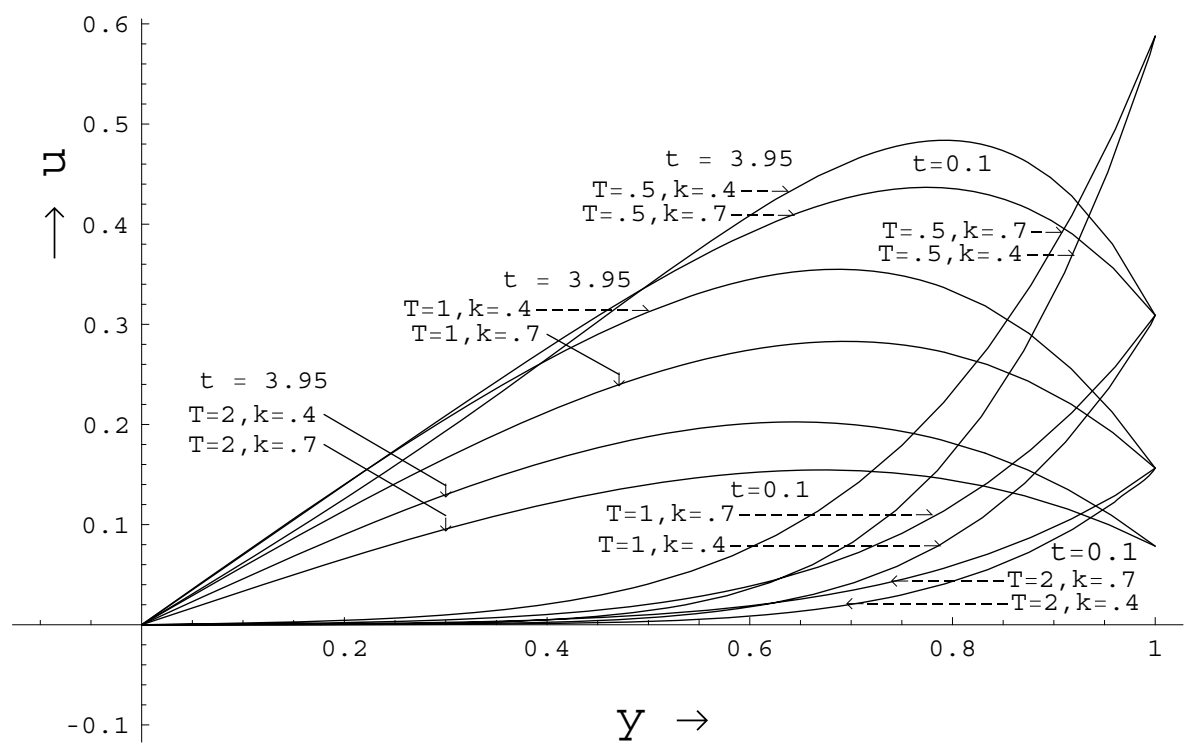

Figure 4 - Effect of the elasticity $(k)$ on the fluid velocity for different values of time period $(T)$ when $M=1.0$.

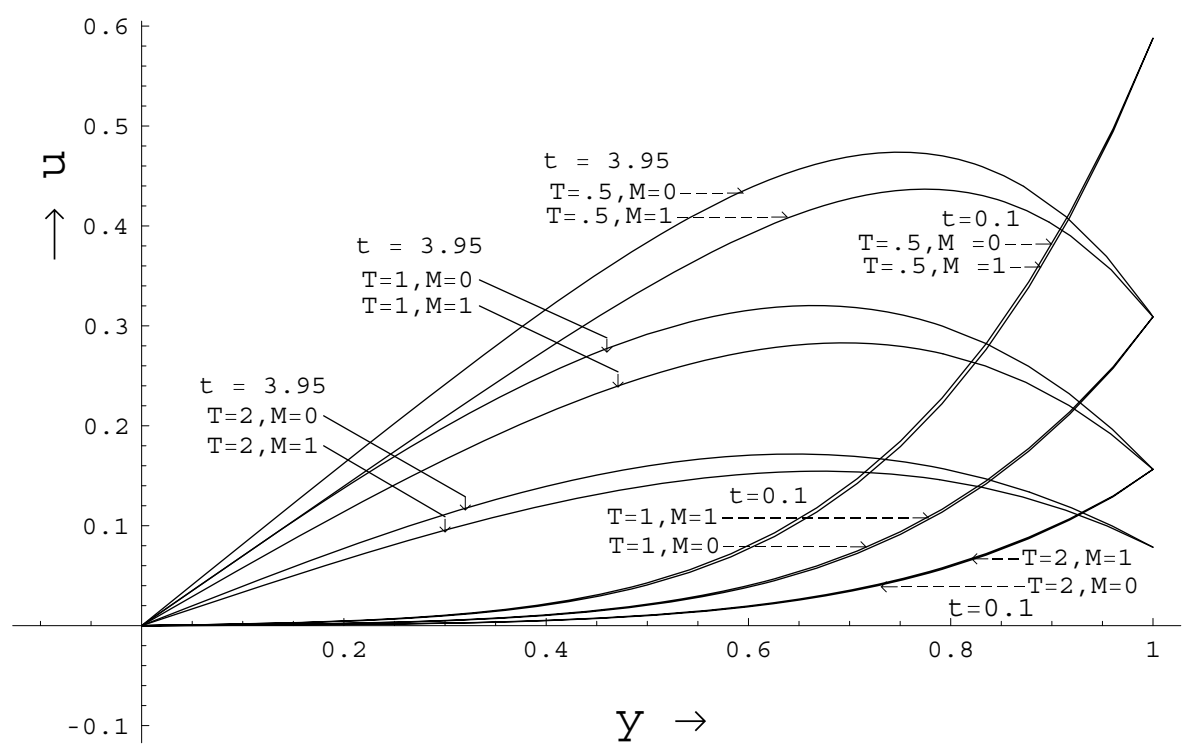

Figure 5 - Effect of the magnetic field $(M)$ on the fluid velocity for different values of time period $(T)$ when $k=0.7$. 
$(T)$ while Figure 5 reflects the enhancement of damping effect on the flows produced by the magnetic field with the increase of time period $(T)$. It is to be mentioned here that in all the numerical calculations mentioned above the distance between the plates is taken as $d=1$ and the non-zero values of the various dimensionless parameters $M, T, t, k$ and $d$ are taken arbitrarily just to test the methodology.

The fluctuations of the skin-friction with time on the lower and the upper plate are shown in Figures $6(\mathrm{a}, \mathrm{b}, \mathrm{c})$ and $7(\mathrm{a}, \mathrm{b}, \mathrm{c})$ respectively for different $a \mathrm{rbi}$ trary non-zero values of $M, k$ and $T$. The results are also presented in Tables 3 and 4. It is noticed that the amplitude of skin-friction increases with the magnetic field at the upper plate and diminishes with the same at the lower plate. Additionally, the effect of the elasticity $(k)$ on the skin-friction decreases with the increase of the magnetic field at the lower plate while a reverse effect is found at the upper plate. In general, for fixed values of $M$ and $T$ the amplitude of skin-friction at the lower plate increases with elastic parameter $(k)$ when the flow is developing and decreases with the same when the flow is retarding. A similar effect is also observed on the upper plate. Finally, the skin-friction on the plates corresponding to hydrodynamic situation are represented by the curves $M=0, k=1$ in Figures $6(\mathrm{a}, \mathrm{b}, \mathrm{c})$ and $7(\mathrm{a}, \mathrm{b}, \mathrm{c})$. It appears from these figures that the amplitude of skin-friction at the lower plate due to viscoelastic fluids remains always less than its classical value while at the upper plate no such definite conclusion can be made.

\section{Conclusion}

An initial-boundary value problem is solved for the motion of an incompressible, electrically conducting, viscoelastic Oldroyd-B fluid confined in a channel bounded by two infinite rigid non-conducting parallel plates in presence of an external magnetic field when the flow is generated impulsively from rest due to multiple frequency unidirectional motion of the upper plate with the lower plate held fixed. A similar type of problem in which the upper plate started impulsively from rest with velocity tooth pulses subjected on the upper plate with the lower plate kept stationary has already been solved in [13]. Thus in the above two problems, the boundary conditions responsible for generating the 


\begin{tabular}{|c|c|c|c|c|c|c|c|c|c|c|c|c|}
\hline$t$ & $T$ & $M$ & $k / y$ & 0.0 & 0.125 & 0.25 & 0.375 & 0.5 & 0.625 & 0.75 & 0.875 & 1.0 \\
\hline \multirow{27}{*}{0.125} & \multirow{9}{*}{0.5} & \multirow{3}{*}{0.0} & 0.4 & 0.0 & 0.0008 & 0.0031 & 0.0105 & 0.0309 & 0.0805 & 0.1860 & 0.3830 & 0.7071 \\
\hline & & & 0.7 & 0.0 & 0.0059 & 0.0160 & 0.0360 & 0.0744 & 0.1435 & 0.2595 & 0.4408 & 0.7071 \\
\hline & & & 1.0 & 0.0 & 0.0137 & 0.0327 & 0.0631 & 0.1124 & 0.1900 & 0.3068 & 0.4747 & 0.7071 \\
\hline & & \multirow{3}{*}{1.0} & 0.4 & 0.0 & 0.0007 & 0.0029 & 0.0097 & 0.0289 & 0.0762 & 0.1785 & 0.3742 & 0.7071 \\
\hline & & & 0.7 & 0.0 & 0.0055 & 0.0149 & 0.0337 & 0.0702 & 0.1370 & 0.2509 & 0.4328 & 0.7071 \\
\hline & & & 1.0 & 0.0 & 0.0128 & 0.0307 & 0.0595 & 0.1069 & 0.1824 & 0.2980 & 0.4673 & 0.7071 \\
\hline & & \multirow{3}{*}{5.0} & 0.4 & 0.0 & 0.0001 & 0.0004 & 0.0018 & 0.0067 & 0.0234 & 0.0767 & 0.2382 & 0.7071 \\
\hline & & & 0.7 & 0.0 & 0.0010 & 0.0029 & 0.0080 & 0.0209 & 0.0524 & 0.1276 & 0.3031 & 0.7071 \\
\hline & & & 1.0 & 0.0 & 0.0027 & 0.0073 & 0.0168 & 0.0370 & 0.0792 & 0.1665 & 0.3448 & 0.7071 \\
\hline & \multirow{9}{*}{1.0} & \multirow{3}{*}{0.0} & 0.4 & 0.0 & 0.0004 & 0.0016 & 0.0052 & 0.0156 & 0.0409 & 0.0958 & 0.2011 & 0.3827 \\
\hline & & & 0.7 & 0.0 & 0.0030 & 0.0080 & 0.0181 & 0.0378 & 0.0736 & 0.1348 & 0.2328 & 0.3827 \\
\hline & & & 1.0 & 0.0 & 0.0069 & 0.0165 & 0.0320 & 0.0575 & 0.0981 & 0.1602 & 0.2514 & 0.3827 \\
\hline & & \multirow{3}{*}{1.0} & 0.4 & 0.0 & 0.0004 & 0.0014 & 0.0048 & 0.0146 & 0.0387 & 0.0920 & 0.1966 & 0.3827 \\
\hline & & & 0.7 & 0.0 & 0.0027 & 0.0074 & 0.0170 & 0.0357 & 0.0703 & 0.1304 & 0.2286 & 0.3827 \\
\hline & & & 1.0 & 0.0 & 0.0064 & 0.0155 & 0.0302 & 0.0547 & 0.0942 & 0.1557 & 0.2476 & 0.3827 \\
\hline & & \multirow{3}{*}{5.0} & 0.4 & 0.0 & 0.0001 & 0.0002 & 0.0009 & 0.0034 & 0.0120 & 0.0399 & 0.1259 & 0.3827 \\
\hline & & & 0.7 & 0.0 & 0.0005 & 0.0015 & 0.0041 & 0.0107 & 0.0272 & 0.0669 & 0.1610 & 0.3827 \\
\hline & & & 1.0 & 0.0 & 0.0014 & 0.0037 & 0.0086 & 0.0191 & 0.0413 & 0.0877 & 0.1836 & 0.3827 \\
\hline & \multirow{9}{*}{2.0} & \multirow{3}{*}{0.0} & 0.4 & 0.0 & 0.0002 & 0.0007 & 0.0025 & 0.0076 & 0.0202 & 0.0477 & 0.1010 & 0.1950 \\
\hline & & & 0.7 & 0.0 & 0.0014 & 0.0039 & 0.0089 & 0.0187 & 0.0366 & 0.0674 & 0.1170 & 0.1950 \\
\hline & & & 1.0 & 0.0 & 0.0034 & 0.0081 & 0.0158 & 0.0285 & 0.0488 & 0.0802 & 0.1265 & 0.1950 \\
\hline & & \multirow{3}{*}{1.0} & 0.4 & 0.0 & 0.0002 & 0.0007 & 0.0024 & 0.0072 & 0.0192 & 0.0458 & 0.0987 & 0.1950 \\
\hline & & & 0.7 & 0.0 & 0.0013 & 0.0036 & 0.0083 & 0.0176 & 0.0350 & 0.0652 & 0.1150 & 0.1950 \\
\hline & & & 1.0 & 0.0 & 0.0032 & 0.0076 & 0.0149 & 0.0271 & 0.0469 & 0.0779 & 0.1246 & 0.1950 \\
\hline & & \multirow{3}{*}{5.0} & 0.4 & 0.0 & 0.0 & 0.0001 & 0.0004 & 0.0017 & 0.0060 & 0.0200 & 0.0634 & 0.1950 \\
\hline & & & 0.7 & 0.0 & 0.0002 & 0.0007 & 0.0020 & 0.0053 & 0.0136 & 0.0335 & 0.0811 & 0.1950 \\
\hline & & & 1.0 & 0.0 & 0.0007 & 0.0018 & 0.0043 & 0.0095 & 0.0207 & 0.0440 & 0.0926 & 0.1950 \\
\hline
\end{tabular}

Table 1 - The fluid velocity $u$ corresponding to developing flow for different values of $T, M, k$.

\begin{tabular}{|c|c|c|c|c|c|c|c|c|c|c|c|c|}
\hline$t$ & $T$ & $M$ & $k / y$ & 0.0 & 0.125 & 0.25 & 0.375 & 0.5 & 0.625 & 0.75 & 0.875 & 1.0 \\
\hline \multirow{27}{*}{3.85} & \multirow{9}{*}{0.5} & \multirow{3}{*}{0.0} & 0.4 & 0.0 & 0.0743 & 0.1549 & 0.2481 & 0.3592 & 0.4895 & 0.6299 & 0.7539 & 0.8090 \\
\hline & & & 0.7 & 0.0 & 0.0927 & 0.1899 & 0.2951 & 0.4095 & 0.5306 & 0.6503 & 0.7521 & 0.8090 \\
\hline & & & 1.0 & 0.0 & 0.1051 & 0.2124 & 0.3232 & 0.4372 & 0.5518 & 0.6604 & 0.7518 & 0.8090 \\
\hline & & \multirow{3}{*}{1.0} & 0.4 & 0.0 & 0.0646 & 0.1356 & 0.2197 & 0.3232 & 0.4487 & 0.5907 & 0.7270 & 0.8090 \\
\hline & & & 0.7 & 0.0 & 0.0807 & 0.1664 & 0.2615 & 0.3685 & 0.4868 & 0.6105 & 0.7261 & 0.8090 \\
\hline & & & 1.0 & 0.0 & 0.0915 & 0.1862 & 0.2865 & 0.3936 & 0.5062 & 0.6201 & 0.7260 & 0.8090 \\
\hline & & \multirow{3}{*}{5.0} & 0.4 & 0.0 & 0.0058 & 0.0143 & 0.0294 & 0.0585 & 0.1153 & 0.2259 & 0.4354 & 0.8090 \\
\hline & & & 0.7 & 0.0 & 0.0071 & 0.0173 & 0.0350 & 0.0678 & 0.1288 & 0.2416 & 0.4464 & 0.8090 \\
\hline & & & 1.0 & 0.0 & 0.0087 & 0.0207 & 0.0407 & 0.0760 & 0.1393 & 0.2525 & 0.4539 & 0.8090 \\
\hline & \multirow{9}{*}{1.0} & \multirow{3}{*}{0.0} & 0.4 & 0.0 & 0.1147 & 0.2273 & 0.3346 & 0.4305 & 0.5056 & 0.5465 & 0.5360 & 0.4540 \\
\hline & & & 0.7 & 0.0 & 0.1073 & 0.2111 & 0.3073 & 0.3911 & 0.4564 & 0.4953 & 0.4982 & 0.4540 \\
\hline & & & 1.0 & 0.0 & 0.0983 & 0.1933 & 0.2815 & 0.3589 & 0.4209 & 0.4619 & 0.4754 & 0.4540 \\
\hline & & \multirow{3}{*}{1.0} & 0.4 & 0.0 & 0.0951 & 0.1901 & 0.2835 & 0.3718 & 0.4474 & 0.4987 & 0.5085 & 0.4540 \\
\hline & & & 0.7 & 0.0 & 0.0897 & 0.1777 & 0.2620 & 0.3393 & 0.4051 & 0.4527 & 0.4731 & 0.4540 \\
\hline & & & 1.0 & 0.0 & 0.0828 & 0.1640 & 0.2416 & 0.3131 & 0.3753 & 0.4236 & 0.4524 & 0.4540 \\
\hline & & \multirow{3}{*}{5.0} & 0.4 & 0.0 & 0.0060 & 0.0145 & 0.0291 & 0.0554 & 0.1024 & 0.1823 & 0.3054 & 0.4540 \\
\hline & & & 0.7 & 0.0 & 0.0061 & 0.0146 & 0.0283 & 0.0520 & 0.0931 & 0.1627 & 0.2769 & 0.4540 \\
\hline & & & 1.0 & 0.0 & 0.0058 & 0.0137 & 0.0262 & 0.0475 & 0.0846 & 0.1492 & 0.2611 & 0.4540 \\
\hline & \multirow{9}{*}{2.0} & \multirow{3}{*}{0.0} & 0.4 & 0.0 & 0.0850 & 0.1644 & 0.2328 & 0.2849 & 0.3155 & 0.3199 & 0.2938 & 0.2334 \\
\hline & & & 0.7 & 0.0 & 0.0671 & 0.1306 & 0.1871 & 0.2329 & 0.2642 & 0.2774 & 0.2684 & 0.2334 \\
\hline & & & 1.0 & 0.0 & 0.0582 & 0.1138 & 0.1640 & 0.2060 & 0.2371 & 0.2541 & 0.2540 & 0.2334 \\
\hline & & \multirow{3}{*}{1.0} & 0.4 & 0.0 & 0.0715 & 0.1396 & 0.2005 & 0.2503 & 0.2843 & 0.2971 & 0.2825 & 0.2334 \\
\hline & & & 0.7 & 0.0 & 0.0565 & 0.1109 & 0.1607 & 0.2034 & 0.2360 & 0.2549 & 0.2557 & 0.2334 \\
\hline & & & 1.0 & 0.0 & 0.0483 & 0.0951 & 0.1387 & 0.1773 & 0.2088 & 0.2307 & 0.2401 & 0.2334 \\
\hline & & \multirow{3}{*}{5.0} & 0.4 & 0.0 & 0.0059 & 0.0142 & 0.0275 & 0.0500 & 0.0864 & 0.1402 & 0.2042 & 0.2334 \\
\hline & & & 0.7 & 0.0 & 0.0049 & 0.0113 & 0.0213 & 0.0374 & 0.0635 & 0.1039 & 0.1620 & 0.2334 \\
\hline & & & 1.0 & 0.0 & 0.0031 & 0.0073 & 0.0139 & 0.0251 & 0.0443 & 0.0776 & 0.1350 & 0.2334 \\
\hline
\end{tabular}

Table 2 - The fluid velocity $u$ corresponding to retarding flow for different values of $T, M, k$. 


\begin{tabular}{|c|c|c|c|c|c|c|c|c|c|c|c|c|c|}
\hline$T$ & $M$ & $k / t$ & 0.0 & 0.2 & 0.4 & 0.6 & 0.8 & 1.0 & 1.2 & 1.4 & 1.6 & 1.8 & 2.0 \\
\hline \multirow{9}{*}{0.5} & \multirow{3}{*}{0.0} & 0.4 & 0.0 & 0.024 & 0.249 & 0.411 & 0.421 & 0.556 & 0.508 & 0.557 & 0.598 & 0.536 & 0.632 \\
\hline & & 0.7 & 0.0 & 0.150 & 0.572 & 0.443 & 0.547 & 0.665 & 0.453 & 0.706 & 0.520 & 0.602 & 0.708 \\
\hline & & 1.0 & 0.0 & 0.441 & 0.929 & 0.452 & 0.806 & 0.747 & 0.521 & 0.896 & 0.411 & 0.770 & 0.718 \\
\hline & \multirow{3}{*}{1.0} & 0.4 & 0.0 & 0.021 & 0.204 & 0.319 & 0.326 & 0.440 & 0.400 & 0.456 & 0.488 & 0.446 & 0.530 \\
\hline & & 0.7 & 0.0 & 0.135 & 0.486 & 0.351 & 0.459 & 0.550 & 0.374 & 0.598 & 0.425 & 0.514 & 0.594 \\
\hline & & 1.0 & 0.0 & 0.389 & 0.797 & 0.359 & 0.695 & 0.622 & 0.442 & 0.768 & 0.327 & 0.669 & 0.600 \\
\hline & \multirow{3}{*}{5.0} & 0.4 & 0.0 & 0.001 & 0.006 & 0.007 & 0.010 & 0.014 & 0.015 & 0.020 & 0.021 & 0.024 & 0.027 \\
\hline & & 0.7 & 0.0 & 0.014 & 0.026 & 0.013 & 0.031 & 0.027 & 0.028 & 0.038 & 0.024 & 0.041 & 0.035 \\
\hline & & 1.0 & 0.0 & 0.046 & 0.060 & 0.015 & 0.065 & 0.032 & 0.046 & 0.060 & 0.014 & 0.065 & 0.032 \\
\hline \multirow{9}{*}{1.0} & \multirow{3}{*}{0.0} & 0.4 & 0.0 & 0.012 & 0.151 & 0.388 & 0.581 & 0.613 & 0.472 & 0.435 & 0.558 & 0.685 & 0.684 \\
\hline & & 0.7 & 0.0 & 0.078 & 0.396 & 0.682 & 0.753 & 0.559 & 0.323 & 0.512 & 0.754 & 0.806 & 0.600 \\
\hline & & 1.0 & 0.0 & 0.238 & 0.700 & 0.971 & 0.895 & 0.492 & 0.291 & 0.682 & 0.948 & 0.875 & 0.476 \\
\hline & \multirow{3}{*}{1.0} & 0.4 & 0.0 & 0.011 & 0.125 & 0.310 & 0.453 & 0.473 & 0.368 & 0.360 & 0.469 & 0.568 & 0.560 \\
\hline & & 0.7 & 0.0 & 0.071 & 0.339 & 0.570 & 0.618 & 0.449 & 0.265 & 0.441 & 0.641 & 0.673 & 0.493 \\
\hline & & 1.0 & 0.0 & 0.208 & 0.608 & 0.831 & 0.753 & 0.398 & 0.243 & 0.594 & 0.814 & 0.739 & 0.386 \\
\hline & \multirow{3}{*}{5.0} & 0.4 & 0.0 & 0.001 & 0.004 & 0.008 & 0.012 & 0.014 & 0.015 & 0.018 & 0.022 & 0.026 & 0.027 \\
\hline & & 0.7 & 0.0 & 0.007 & 0.021 & 0.031 & 0.031 & 0.022 & 0.021 & 0.034 & 0.041 & 0.040 & 0.030 \\
\hline & & 1.0 & 0.0 & 0.025 & 0.057 & 0.067 & 0.052 & 0.017 & 0.025 & 0.057 & 0.067 & 0.052 & 0.017 \\
\hline \multirow{9}{*}{2.0} & \multirow{3}{*}{0.0} & 0.4 & 0.0 & 0.006 & 0.079 & 0.224 & 0.400 & 0.570 & 0.702 & 0.776 & 0.781 & 0.716 & 0.584 \\
\hline & & 0.7 & 0.0 & 0.039 & 0.213 & 0.428 & 0.624 & 0.771 & 0.850 & 0.850 & 0.772 & 0.621 & 0.413 \\
\hline & & 1.0 & 0.0 & 0.119 & 0.382 & 0.641 & 0.843 & 0.966 & 0.995 & 0.929 & 0.772 & 0.512 & 0.258 \\
\hline & \multirow{3}{*}{1.0} & 0.4 & 0.0 & 0.005 & 0.065 & 0.180 & 0.316 & 0.447 & 0.552 & 0.614 & 0.625 & 0.582 & 0.488 \\
\hline & & 0.7 & 0.0 & 0.035 & 0.183 & 0.360 & 0.520 & 0.639 & 0.703 & 0.703 & 0.640 & 0.516 & 0.346 \\
\hline & & 1.0 & 0.0 & 0.104 & 0.334 & 0.554 & 0.724 & 0.825 & 0.846 & 0.786 & 0.650 & 0.450 & 0.207 \\
\hline & \multirow{3}{*}{5.0} & 0.4 & 0.0 & 0.0 & 0.002 & 0.005 & 0.008 & 0.013 & 0.017 & 0.022 & 0.025 & 0.027 & 0.028 \\
\hline & & 0.7 & 0.0 & 0.004 & 0.012 & 0.021 & 0.029 & 0.036 & 0.040 & 0.041 & 0.039 & 0.034 & 0.027 \\
\hline & & 1.0 & 0.0 & 0.013 & 0.032 & 0.049 & 0.061 & 0.067 & 0.066 & 0.059 & 0.046 & 0.029 & 0.008 \\
\hline
\end{tabular}

Table 3 - The skin-friction $\tau_{0}$ at the lower plate $y=0$ for different values of $T, M, k$.

\begin{tabular}{|c|c|c|c|c|c|c|c|c|c|c|c|c|c|}
\hline$T$ & $M$ & $k / t$ & 0.0 & 0.2 & 0.4 & 0.6 & 0.8 & 1.0 & 1.2 & 1.4 & 1.6 & 1.8 & 2.0 \\
\hline \multirow{9}{*}{0.5} & \multirow{3}{*}{0.0} & 0.4 & 0.0 & 1.389 & -0.150 & 1.059 & 0.716 & -0.979 & 1.278 & -0.129 & 1.141 & 0.820 & -0.876 \\
\hline & & 0.7 & 0.0 & 1.698 & -0.345 & 1.368 & 0.833 & -1.317 & 1.592 & -0.316 & 1.425 & 0.888 & -1.270 \\
\hline & & 1.0 & 0.0 & 1.862 & -0.540 & 1.583 & 0.869 & -1.627 & 1.794 & -0.497 & 1.632 & 0.911 & -1.593 \\
\hline & \multirow{4}{*}{1.0} & 0.4 & 0.0 & 1.504 & 0.0307 & 1.219 & 0.959 & -0.796 & 1.498 & 0.104 & 1.316 & 1.057 & $\begin{array}{c}-0.708 \\
\end{array}$ \\
\hline & & 0.7 & 0.0 & 1.844 & -0.134 & 1.525 & 1.079 & -1.158 & 1.802 & -0.084 & 1.584 & 1.132 & -1.113 \\
\hline & & 1.0 & 0.0 & 2.054 & -0.289 & 1.747 & 1.139 & -1.470 & 2.013 & -0.251 & 1.785 & 1.172 & -1.443 \\
\hline & & 0.4 & 0.0 & 3.421 & 2.112 & 2.739 & 3.722 & 0.511 & 4.044 & 2.593 & 3.113 & 4.012 & 0.738 \\
\hline & \multirow[t]{2}{*}{5.0} & 0.7 & 0.0 & 4.266 & 2.270 & 3.114 & 4.175 & -0.055 & 4.542 & 2.492 & 3.292 & 4.318 & 0.060 \\
\hline & & 1.0 & 0.0 & 4.966 & 2.443 & 3.446 & 4.590 & -0.471 & 4.978 & 2.452 & 3.454 & 4.596 & -0.466 \\
\hline \multirow{9}{*}{1.0} & \multirow{4}{*}{0.0} & 0.4 & 0.0 & 0.984 & 1.066 & 0.668 & -0.007 & -0.602 & 0.906 & 1.107 & 0.763 & 0.104 & $\begin{array}{l}-0.497 \\
\end{array}$ \\
\hline & & 0.7 & 0.0 & 1.221 & 1.246 & 0.722 & -0.083 & -0.752 & 1.172 & 1.291 & 0.782 & -0.028 & -0.706 \\
\hline & & 1.0 & 0.0 & 1.363 & 1.379 & 0.788 & -0.132 & -0.906 & 1.317 & 1.403 & 0.816 & -0.108 & -0.887 \\
\hline & & 0.4 & 0.0 & 1.050 & 1.233 & 0.912 & 0.246 & -0.427 & 1.072 & 1.319 & 1.015 & 0.346 & $\begin{array}{l}-0.338 \\
\end{array}$ \\
\hline & \multirow[t]{3}{*}{1.0} & 0.7 & 0.0 & 1.304 & 1.449 & 0.995 & 0.166 & -0.620 & 1.306 & 1.508 & 1.056 & 0.219 & -0.576 \\
\hline & & 1.0 & 0.0 & 1.474 & 1.620 & 1.087 & 0.119 & -0.795 & 1.447 & 1.641 & 1.109 & 0.137 & -0.780 \\
\hline & & 0.4 & 0.0 & 2.170 & 3.537 & 3.714 & 2.598 & 0.657 & 2.780 & 4.008 & 4.080 & 2.883 & 0.880 \\
\hline & \multirow[t]{2}{*}{5.0} & 0.7 & 0.0 & 2.725 & 4.242 & 4.204 & 2.612 & 0.160 & 2.997 & 4.461 & 4.379 & 2.753 & 0.273 \\
\hline & & 1.0 & 0.0 & 3.183 & 4.849 & 4.658 & 2.683 & -0.207 & 3.192 & 4.857 & 4.664 & 2.688 & -0.202 \\
\hline \multirow{9}{*}{2.0} & \multirow{4}{*}{0.0} & 0.4 & 0.0 & 0.531 & 0.746 & 0.849 & 0.859 & 0.787 & 0.648 & 0.457 & 0.232 & -0.004 & -0.183 \\
\hline & & 0.7 & 0.0 & 0.660 & 0.890 & 0.994 & 0.997 & 0.909 & 0.738 & 0.501 & 0.219 & -0.079 & -0.301 \\
\hline & & 1.0 & 0.0 & 0.740 & 1.001 & 1.130 & 1.141 & 1.037 & 0.829 & 0.538 & 0.193 & -0.172 & -0.442 \\
\hline & & 0.4 & 0.0 & 0.565 & 0.841 & 1.018 & 1.096 & 1.076 & 0.961 & 0.762 & 0.499 & 0.195 & $\begin{array}{l}-0.072 \\
\end{array}$ \\
\hline & \multirow[t]{3}{*}{1.0} & 0.7 & 0.0 & 0.703 & 1.006 & 1.187 & 1.254 & 1.205 & 1.044 & 0.787 & 0.457 & 0.087 & -0.223 \\
\hline & & 1.0 & 0.0 & 0.798 & 1.139 & 1.345 & 1.413 & 1.341 & 1.136 & 0.818 & 0.419 & -0.021 & -0.382 \\
\hline & & 0.4 & 0.0 & 1.144 & 2.198 & 3.119 & 3.798 & 4.156 & 4.146 & 3.759 & 3.029 & 2.020 & 0.879 \\
\hline & \multirow[t]{2}{*}{5.0} & 0.7 & 0.0 & 1.438 & 2.661 & 3.656 & 4.322 & 4.586 & 4.418 & 3.833 & 2.884 & 1.661 & 0.351 \\
\hline & & 1.0 & 0.0 & 1.680 & 3.056 & 4.130 & 4.798 & 4.996 & 4.704 & 3.951 & 2.810 & 1.394 & -0.078 \\
\hline
\end{tabular}

Table 4 - The skin-friction $\tau_{d}$ at the upper plate $y=d$ for different values of $T, M, k$. 


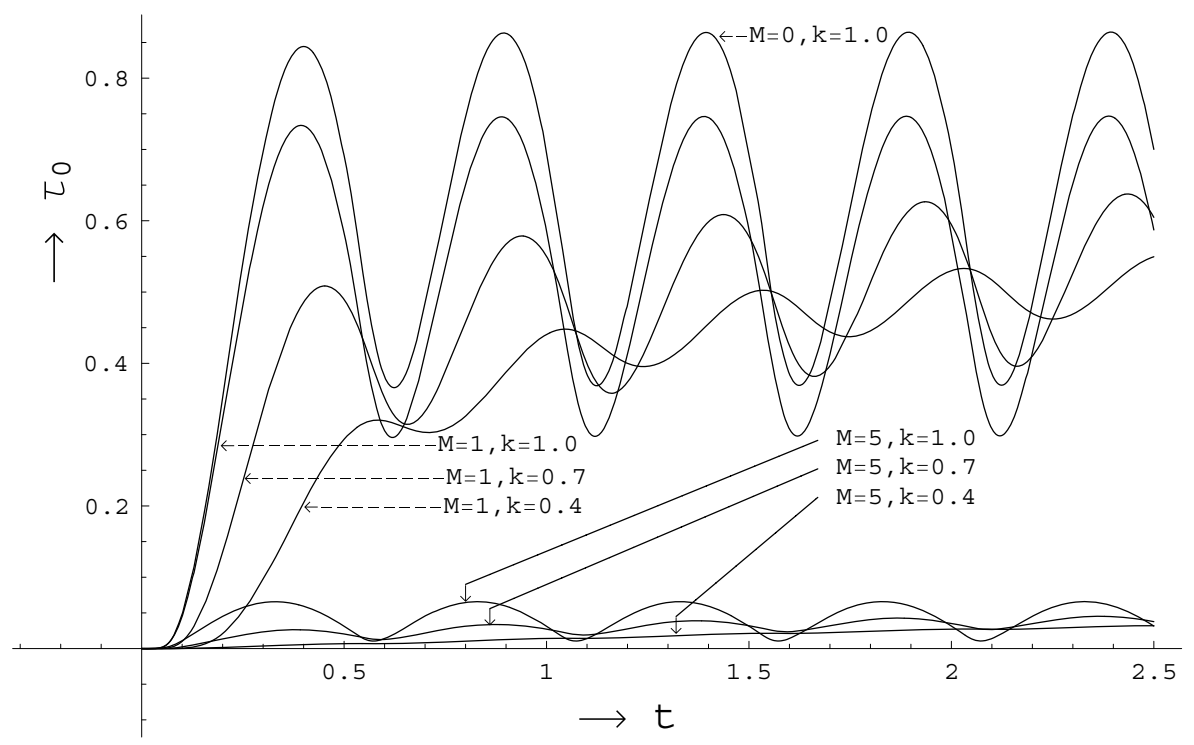

Figure 6(a) - Effects of the magnetic field $(M)$ and the elasticity $(k)$ on the skin-friction at the lower plate $y=0$ when $T=0.5$.

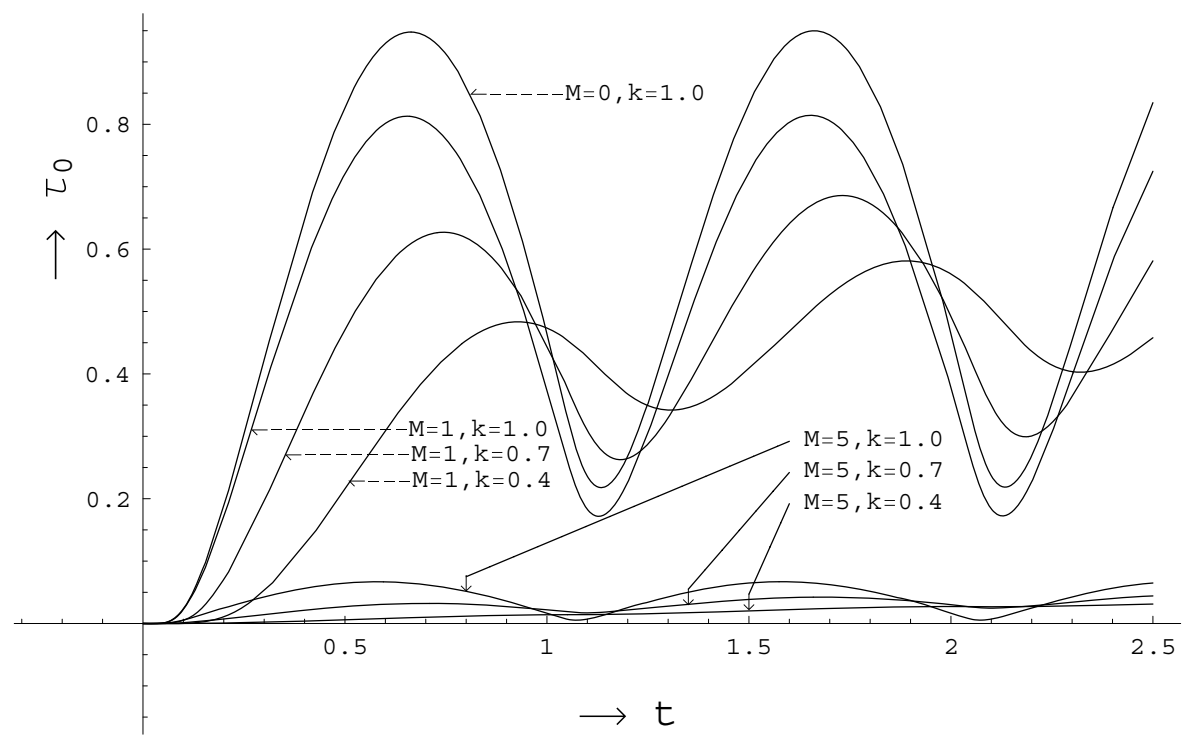

Figure 6(b) - Effects of the magnetic field $(M)$ and the elasticity $(k)$ on the skin-friction at the lower plate $y=0$ when $T=1.0$. 


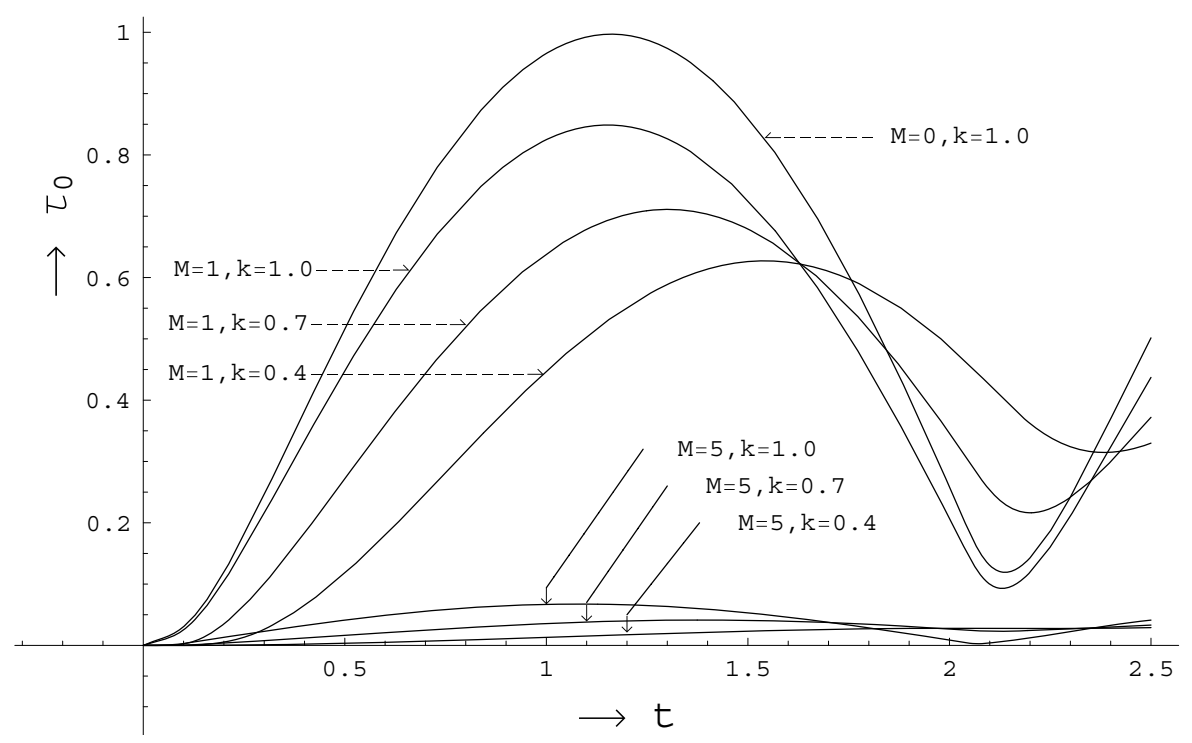

Figure 6(c) - Effects of the magnetic field $(M)$ and the elasticity $(k)$ on the skin-friction at the lower plate $y=0$ when $T=2.0$.

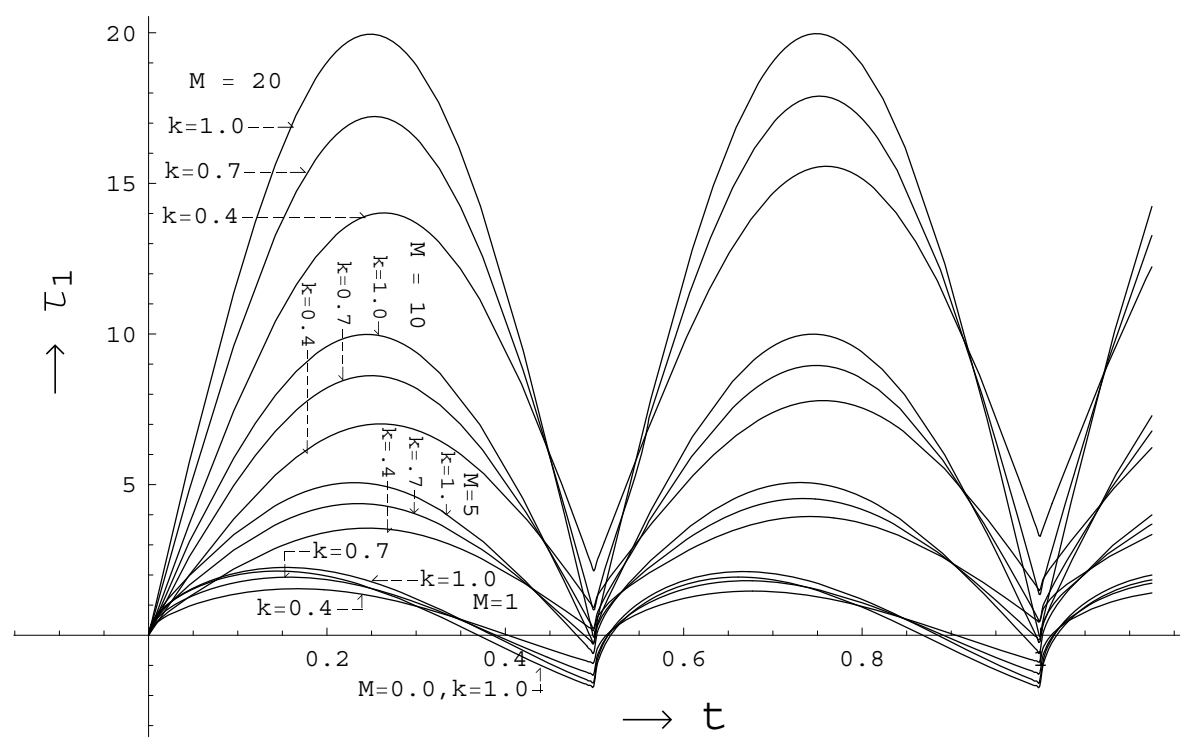

Figure 7(a) - Effects of the magnetic field $(M)$ and the elasticity $(k)$ on the skin-friction at the upper plate $y=d$ when $T=0.5$. 


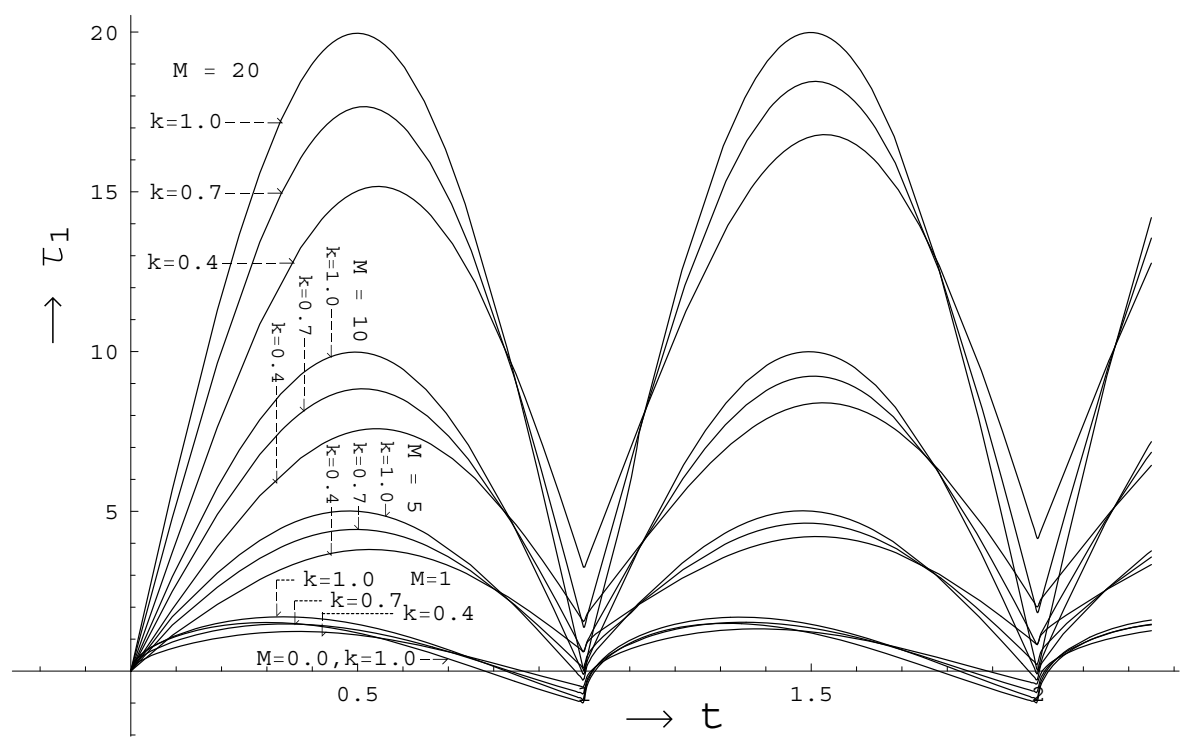

Figure 7(b) - Effects of the magnetic field (M) and the elasticity $(k)$ on the skin-friction at the upper plate $y=d$ when $T=1.0$.

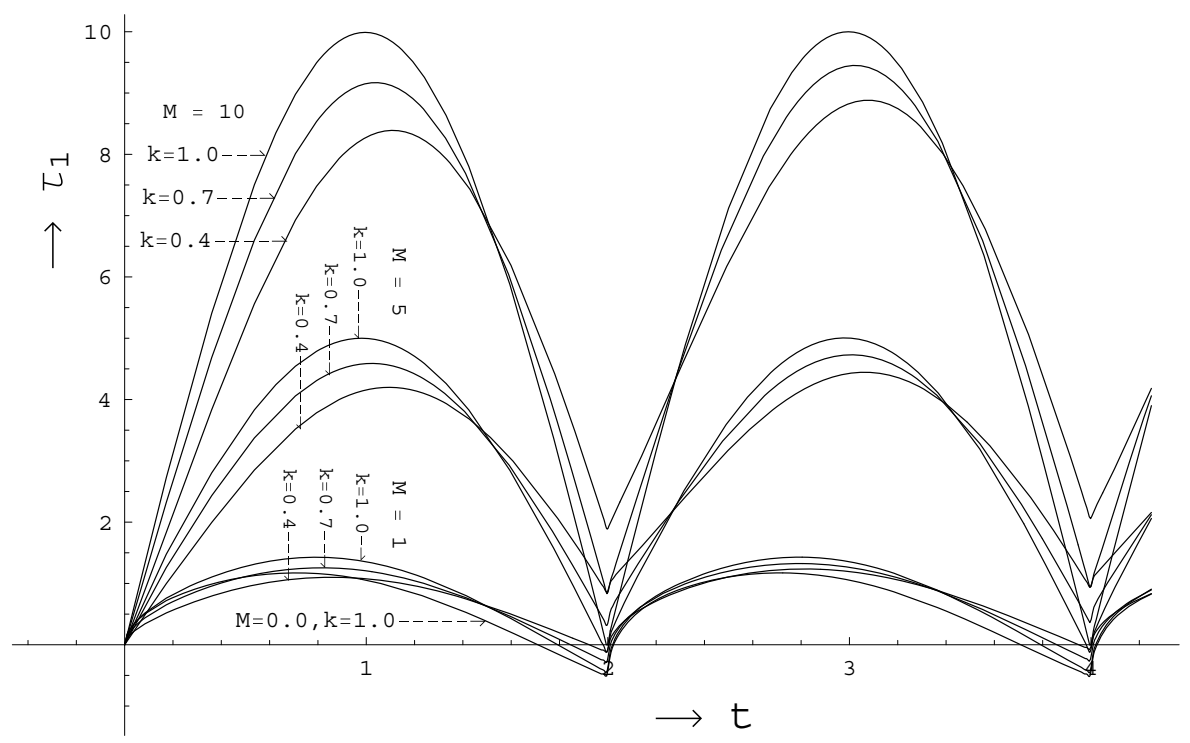

Figure 7(c) - Effects of the magnetic field $(M)$ and the elasticity (k) on the skin-friction at the upper plate $y=d$ when $T=2.0$. 
unsteady motion differ significantly. As a result, the flow phenomena discussed in [13] is not the same as that of the present analysis. Moreover, the fluid flow, generated by tooth pulses as discussed in [13], is supposed to have importance in white dwarf asteroseismology [14] and acoustic and acousto-gravity wave pulses caused by sources of seismic origin [15]. On the other hand, the flow produced by rectified sine pulses as considered in the present paper has applications in planetary tides and sunspot cycles [16], peristaltic transport of a particle-fluid suspension [17], pulsed microwave plasma etching of polymers [18], adaptive noise reduction for pulmonary artery blood pressure [19] and Wavelab's elevated frequency resolution to music therapy [20]. Several other applications of tooth pulses and rectified sine pulses in physical problems may also be found in the literature as substantial references.

We further observe that the skin-friction on the lower plate appears to have symmetrical peaks for all values of the magnetic field $M$ while the skin-friction on the upper plate contains non-symmetrical peaks particularly at small values of $M$ due to the appearance of negative skin-friction in such a stage. However, with the increase of the strength of the magnetic field the values of the negative skin-friction on the upper plate, developed during its retarding motion, goes on diminishing. This leads to the restoration of symmetrical peaks of skin-friction on the upper plate for large values of $M$ when skin-friction remains always positive (Figures $7(a, b, c)$ ). This is a consequence of the effect of magnetic field in presence of pulsation.

Finally, we add that in order to produce a four digit accuracy in Tables 1 to 4 , we have considered first 100 terms of $m$ and $n$ series. For the sake of convenience in numerical calculation two different expansion indexes $m$ and $n$ are considered in Eq.(4.13) and Eq.(4.27). However one can take only one expansion index to represent both the series.

Acknowledgement. The authors are very much grateful to the referees for providing many helpful suggestions to improve the paper in its present form.

\section{Appendix}

Let $S_{t}$ denotes the transient part of the solution of the equation [4.13]. 
Then we have

$$
S_{t}=\sum_{n=1}^{\infty}\left\{W_{n}^{\prime}(0) \frac{e^{m_{1} t}-e^{m_{2} t}}{m_{1}-m_{2}}+W_{n}(0) \frac{m_{1} e^{m_{2} t}-m_{2} e^{m_{1} t}}{m_{1}-m_{2}}\right\} \sin \frac{n \pi y}{d}
$$

Now,

$$
\begin{aligned}
& W_{n}^{\prime}(0)=\frac{8 n(-1)^{n}}{d^{2}} \operatorname{Im} \sum_{m=1}^{\infty} \frac{\beta_{m}}{\left\{(2 m)^{2}-1\right\}\left(L_{m}^{2}+\frac{n^{2} \pi^{2}}{d^{2}}\right)} \\
& =\frac{8 n(-1)^{n}}{d^{2}} \operatorname{Im} \sum_{m=1}^{\infty} \frac{\beta_{m}\left(1+i \beta_{m} k\right)}{\left\{(2 m)^{2}-1\right\}\left(s_{1}-i \beta_{m}\right)\left(s_{2}-i \beta_{m}\right)} \\
& =\frac{8 n(-1)^{n}}{d^{2}} \operatorname{Im} \sum_{m=1}^{\infty} \frac{\beta_{m}}{\left\{(2 m)^{2}-1\right\}}\left\{-\frac{1+k s_{1}}{s_{1}-s_{2}} \cdot \frac{1}{s_{1}-i \beta_{m}}+\frac{1+k s_{2}}{s_{1}-s_{2}} \cdot \frac{1}{s_{2}-i \beta_{m}}\right\} \\
& =\frac{8 n(-1)^{n}}{d^{2}} \sum_{m=1}^{\infty} \frac{\beta_{m}}{\left\{(2 m)^{2}-1\right\}}\left\{-\frac{1+k s_{1}}{s_{1}-s_{2}} \cdot \frac{\beta_{m}}{s_{1}^{2}+\beta_{m}^{2}}+\frac{1+k s_{2}}{s_{1}-s_{2}} \cdot \frac{\beta_{m}}{s_{2}^{2}+\beta_{m}^{2}}\right\} \\
& =\frac{8 n(-1)^{n}}{d^{2}} \sum_{m=1}^{\infty}\left\{-\frac{1+k s_{1}}{s_{1}-s_{2}} \cdot \frac{\beta_{m}^{2}}{\left\{(2 m)^{2}-1\right\}\left(s_{1}^{2}+\beta_{m}^{2}\right)}\right. \\
& \left.+\frac{1+k s_{2}}{s_{1}-s_{2}} \cdot \frac{\beta_{m}^{2}}{\left\{(2 m)^{2}-1\right\}\left(s_{2}^{2}+\beta_{m}^{2}\right)}\right\} \\
& =\frac{8 n(-1)^{n}}{d^{2}}\left[\sum_{m=1}^{\infty}-\frac{1+k s_{1}}{s_{1}-s_{2}} \cdot \frac{(2 m)^{2} \pi^{2}}{T^{2}\left\{(2 m)^{2}-1\right\}\left\{s_{1}^{2}+\frac{(2 m)^{2} \pi^{2}}{T^{2}}\right\}}\right. \\
& \left.+\sum_{m=1}^{\infty} \frac{1+k s_{2}}{s_{1}-s_{2}} \cdot \frac{(2 m)^{2} \pi^{2}}{T^{2}\left\{(2 m)^{2}-1\right\}\left\{s_{2}^{2}+\frac{(2 m)^{2} \pi^{2}}{T^{2}}\right\}}\right] \\
& =\frac{8 n(-1)^{n}}{d^{2}}\left[\sum_{m=1}^{\infty}\left(-\frac{1+k s_{1}}{s_{1}-s_{2}}\right)\left\{\frac{1}{(2 m)^{2}-1}-\frac{s_{1}^{2} T^{2}}{\left\{(2 m)^{2}-1\right\}\left\{s_{1}^{2} T^{2}+(2 m)^{2} \pi^{2}\right\}}\right\}\right. \\
& \left.+\sum_{m=1}^{\infty}\left(\frac{1+k s_{2}}{s_{1}-s_{2}}\right)\left\{\frac{1}{(2 m)^{2}-1}-\frac{s_{2}^{2} T^{2}}{\left\{(2 m)^{2}-1\right\}\left\{s_{2}^{2} T^{2}+(2 m)^{2} \pi^{2}\right\}}\right\}\right] \\
& =\frac{8 n(-1)^{n}}{d^{2}} \sum_{m=1}^{\infty}\left[\left\{\frac{1+k s_{2}}{s_{1}-s_{2}}-\frac{1+k s_{1}}{s_{1}-s_{2}}\right\} \frac{1}{(2 m)^{2}-1}\right.
\end{aligned}
$$




$$
\begin{aligned}
& +\frac{1+k s_{1}}{s_{1}-s_{2}}\left\{\frac{1}{(2 m)^{2}-1}-\frac{\pi^{2}}{s_{1}^{2} T^{2}+(2 m)^{2} \pi^{2}}\right\} \frac{s_{1}^{2} T^{2}}{s_{1}^{2} T^{2}+\pi^{2}} \\
& \left.-\frac{1+k s_{2}}{s_{1}-s_{2}}\left\{\frac{1}{(2 m)^{2}-1}-\frac{\pi^{2}}{s_{2}^{2} T^{2}+(2 m)^{2} \pi^{2}}\right\} \frac{s_{2}^{2} T^{2}}{s_{2}^{2} T^{2}+\pi^{2}}\right] \\
& =\frac{8 n(-1)^{n}}{d^{2}}\left[\sum_{m=1}^{\infty} \frac{-k}{(2 m)^{2}-1}+\frac{1}{(2 m)^{2}-1}\left\{\frac{1+k s_{1}}{s_{1}-s_{2}} \frac{s_{1}^{2} T^{2}}{s_{1}^{2} T^{2}+\pi^{2}}\right.\right. \\
& \left.-\frac{1+k s_{2}}{s_{1}-s_{2}} \frac{s_{2}^{2} T^{2}}{s_{2}^{2} T^{2}+\pi^{2}}\right\}-\frac{1+k s_{1}}{s_{1}-s_{2}} \frac{\pi^{2} s_{1}^{2} T^{2}}{s_{1}^{2} T^{2}+\pi^{2}} \sum_{m=1}^{\infty} \frac{1}{s_{1}^{2} T^{2}+(2 m)^{2} \pi^{2}} \\
& \left.+\frac{1+k s_{2}}{s_{1}-s_{2}} \frac{\pi^{2} s_{2}^{2} T^{2}}{s_{2}^{2} T^{2}+\pi^{2}} \sum_{m=1}^{\infty} \frac{1}{s_{2}^{2} T^{2}+(2 m)^{2} \pi^{2}}\right] \\
& =\frac{8 n(-1)^{n}}{d^{2}}\left[\frac{-k}{2}+\frac{1}{2}\left\{\frac{1+k s_{1}}{s_{1}-s_{2}} \frac{s_{1}^{2} T^{2}}{s_{1}^{2} T^{2}+\pi^{2}}-\frac{1+k s_{2}}{s_{1}-s_{2}} \frac{s_{2}^{2} T^{2}}{s_{2}^{2} T^{2}+\pi^{2}}\right\}\right. \\
& -\frac{1+k s_{1}}{s_{1}-s_{2}} \frac{\pi^{2} s_{1}^{2} T^{2}}{s_{1}^{2} T^{2}+\pi^{2}} \frac{1}{4 s_{1} T} \sum_{m=1}^{\infty} \frac{2\left(\frac{s_{1} T}{2}\right)}{\left(\frac{s_{1} T}{2}\right)^{2}+m^{2} \pi^{2}} \\
& \left.+\frac{1+k s_{2}}{s_{1}-s_{2}} \frac{\pi^{2} s_{2}^{2} T^{2}}{s_{2}^{2} T^{2}+\pi^{2}} \frac{1}{4 s_{2} T} \sum_{m=1}^{\infty} \frac{2\left(\frac{s_{2} T}{2}\right)}{\left(\frac{s_{2} T}{2}\right)^{2}+m^{2} \pi^{2}}\right] \\
& =\frac{8 n(-1)^{n}}{d^{2}}\left[\frac{-k}{2}+\frac{1}{2}\left\{\frac{1+k s_{1}}{s_{1}-s_{2}} \frac{s_{1}^{2} T^{2}}{s_{1}^{2} T^{2}+\pi^{2}}-\frac{1+k s_{2}}{s_{1}-s_{2}} \frac{s_{2}^{2} T^{2}}{s_{2}^{2} T^{2}+\pi^{2}}\right\}\right. \\
& -\frac{1+k s_{1}}{4\left(s_{1}-s_{2}\right)} \frac{\pi^{2} s_{1} T}{s_{1}^{2} T^{2}+\pi^{2}}\left\{\operatorname{coth}\left(\frac{s_{1} T}{2}\right)-\frac{2}{s_{1} T}\right\} \\
& \left.+\frac{1+k s_{2}}{4\left(s_{1}-s_{2}\right)} \frac{\pi^{2} s_{2} T}{s_{2}^{2} T^{2}+\pi^{2}}\left\{\operatorname{coth}\left(\frac{s_{2} T}{2}\right)-\frac{2}{s_{2} T}\right\}\right] \\
& =\frac{8 n(-1)^{n}}{d^{2}}\left[\frac{-k}{2}+\frac{1}{2}\left\{\frac{1+k s_{1}}{s_{1}-s_{2}}-\frac{1+k s_{2}}{s_{1}-s_{2}}\right\}\right. \\
& -\frac{1}{2}\left\{\frac{1+k s_{1}}{s_{1}-s_{2}} \frac{\pi^{2}}{s_{1}^{2} T^{2}+\pi^{2}}-\frac{1+k s_{2}}{s_{1}-s_{2}} \frac{\pi^{2}}{s_{2}^{2} T^{2}+\pi^{2}}\right\} \\
& -\frac{1+k s_{1}}{4\left(s_{1}-s_{2}\right)} \frac{\pi^{2} s_{1} T}{s_{1}^{2} T^{2}+\pi^{2}} \operatorname{coth}\left(\frac{s_{1} T}{2}\right)+\frac{1+k s_{2}}{4\left(s_{1}-s_{2}\right)} \frac{\pi^{2} s_{2} T}{s_{2}^{2} T^{2}+\pi^{2}} \operatorname{coth}\left(\frac{s_{2} T}{2}\right)
\end{aligned}
$$




$$
\begin{aligned}
& \left.+\frac{1}{2}\left\{\frac{1+k s_{1}}{s_{1}-s_{2}} \frac{\pi^{2}}{s_{1}^{2} T^{2}+\pi^{2}}-\frac{1+k s_{2}}{s_{1}-s_{2}} \frac{\pi^{2}}{s_{2}^{2} T^{2}+\pi^{2}}\right\}\right] \\
= & \frac{8 n(-1)^{n}}{d^{2}}\left[\frac{-k}{2}+\frac{k}{2}-\frac{1+k s_{1}}{4\left(s_{1}-s_{2}\right)} \frac{\pi^{2} s_{1} T}{s_{1}^{2} T^{2}+\pi^{2}} \operatorname{coth}\left(\frac{s_{1} T}{2}\right)\right. \\
& \left.+\frac{1+k s_{2}}{4\left(s_{1}-s_{2}\right)} \frac{\pi^{2} s_{2} T}{s_{2}^{2} T^{2}+\pi^{2}} \operatorname{coth}\left(\frac{s_{2} T}{2}\right)\right] \\
= & \frac{2 n(-1)^{n+1}}{d^{2}}\left[\frac{1+k s_{1}}{s_{1}-s_{2}} \frac{\pi^{2} s_{1} T}{s_{1}^{2} T^{2}+\pi^{2}} \operatorname{coth}\left(\frac{s_{1} T}{2}\right)\right. \\
& \left.-\frac{1+k s_{2}}{s_{1}-s_{2}} \frac{\pi^{2} s_{2} T}{s_{2}^{2} T^{2}+\pi^{2}} \operatorname{coth}\left(\frac{s_{2} T}{2}\right)\right]
\end{aligned}
$$

Again,

$$
\begin{aligned}
& W_{n}(0)=\frac{4 n(-1)^{n}}{d^{2}}\left[\frac{1}{M^{2}+\frac{n^{2} \pi^{2}}{d^{2}}}-2 \operatorname{Re} \sum_{m=1}^{\infty} \frac{1}{\left\{(2 m)^{2}-1\right\} L_{m}^{2}+\frac{n^{2} \pi^{2}}{d^{2}}}\right] \\
& =\frac{4 n(-1)^{n}}{d^{2}}\left[\frac{1}{M^{2}+\frac{n^{2} \pi^{2}}{d^{2}}}-2 \operatorname{Re} \sum_{m=1}^{\infty} \frac{\left(1+i \beta_{m} k\right)}{\left\{(2 m)^{2}-1\right\}\left(s_{1}-i \beta_{m}\right)\left(s_{2}-i \beta_{m}\right)}\right] \\
& =\frac{4 n(-1)^{n}}{d^{2}}\left[\frac{1}{M^{2}+\frac{n^{2} \pi^{2}}{d^{2}}}-2 \operatorname{Re} \sum_{m=1}^{\infty} \frac{1}{(2 m)^{2}-1} \cdot\left\{-\frac{1+k s_{1}}{s_{1}-s_{2}} \cdot \frac{1}{\left(s_{1}-i \beta_{m}\right)}\right.\right. \\
& \left.\left.+\left(\frac{1+k s_{2}}{s_{1}-s_{2}}\right) \cdot \frac{1}{\left(s_{2}-i \beta_{m}\right)}\right\}\right] \\
& =\frac{4 n(-1)^{n}}{d^{2}}\left[\frac{1}{M^{2}+\frac{n^{2} \pi^{2}}{d^{2}}}-2 \operatorname{Re} \sum_{m=1}^{\infty} \frac{1}{(2 m)^{2}-1} \cdot\left\{-\frac{1+k s_{1}}{s_{1}-s_{2}} \cdot \frac{s_{1}+i \beta_{m}}{\left(s_{1}^{2}+\beta_{m}^{2}\right)}\right.\right. \\
& \left.\left.+\left(\frac{1+k s_{2}}{s_{1}-s_{2}}\right) \cdot \frac{s_{2}+i \beta_{m}}{\left(s_{2}^{2}+\beta_{m}^{2}\right)}\right\}\right] \\
& =\frac{4 n(-1)^{n}}{d^{2}}\left[\frac{1}{M^{2}+\frac{n^{2} \pi^{2}}{d^{2}}}-2 \sum_{m=1}^{\infty}\left\{-\frac{1+k s_{1}}{s_{1}-s_{2}} \cdot \frac{s_{1}}{\left\{(2 m)^{2}-1\right\}\left(s_{1}^{2}+\beta_{m}^{2}\right)}\right.\right. \\
& \left.\left.+\left(\frac{1+k s_{2}}{s_{1}-s_{2}}\right) \cdot \frac{s_{2}}{\left\{(2 m)^{2}-1\right\}\left(s_{2}^{2}+\beta_{m}^{2}\right)}\right\}\right]
\end{aligned}
$$




$$
\begin{aligned}
& =\frac{4 n(-1)^{n}}{d^{2}}\left[\frac{1}{M^{2}+\frac{n^{2} \pi^{2}}{d^{2}}}-2 \sum_{m=1}^{\infty}\left\{-\frac{1+k s_{1}}{s_{1}-s_{2}} \cdot \frac{s_{1} T^{2}}{\left\{(2 m)^{2}-1\right\}\left\{s_{1}^{2} T^{2}+(2 m)^{2} \pi^{2}\right\}}\right.\right. \\
& \left.\left.+\frac{1+k s_{2}}{s_{1}-s_{2}} \cdot \frac{s_{2} T^{2}}{\left\{(2 m)^{2}-1\right\}\left\{s_{2}^{2} T^{2}+(2 m)^{2} \pi^{2}\right\}}\right\}\right] \\
& =\frac{4 n(-1)^{n}}{d^{2}}\left[\frac{1}{M^{2}+\frac{n^{2} \pi^{2}}{d^{2}}}-2 \sum_{m=1}^{\infty}-\left(\frac{1+k s_{1}}{s_{1}-s_{2}}\right)\right. \\
& \times\left\{\frac{1}{(2 m)^{2}-1}-\frac{\pi^{2}}{s_{1}^{2} T^{2}+(2 m)^{2} \pi^{2}}\right\} \frac{s_{1} T^{2}}{s_{1}^{2} T^{2}+\pi^{2}} \\
& \left.+\left(\frac{1+k s_{2}}{s_{1}-s_{2}}\right) \cdot\left\{\frac{1}{(2 m)^{2}-1}-\frac{\pi^{2}}{s_{2}^{2} T^{2}+(2 m)^{2} \pi^{2}}\right\} \frac{s_{2} T^{2}}{s_{2}^{2} T^{2}+\pi^{2}}\right] \\
& =\frac{4 n(-1)^{n}}{d^{2}}\left[\frac{1}{M^{2}+\frac{n^{2} \pi^{2}}{d^{2}}}+\left\{\frac{1+k s_{1}}{s_{1}-s_{2}} \cdot \frac{s_{1} T^{2}}{s_{1}^{2} T^{2}+\pi^{2}}-\frac{1+k s_{2}}{s_{1}-s_{2}} \cdot \frac{s_{2} T^{2}}{s_{2}^{2} T^{2}+\pi^{2}}\right\}\right. \\
& -2\left(\frac{1+k s_{1}}{s_{1}-s_{2}}\right) \cdot \frac{s_{1} T^{2} \pi^{2}}{s_{1}^{2} T^{2}+\pi^{2}} \sum_{m=1}^{\infty} \frac{1}{s_{1}^{2} T^{2}+(2 m)^{2} \pi^{2}} \\
& \left.+2\left(\frac{1+k s_{2}}{s_{1}-s_{2}}\right) \cdot \frac{s_{2} T^{2} \pi^{2}}{s_{2}^{2} T^{2}+\pi^{2}} \sum_{m=1}^{\infty} \frac{1}{s_{2}^{2} T^{2}+(2 m)^{2} \pi^{2}}\right] \\
& =\frac{4 n(-1)^{n}}{d^{2}}\left[\frac{1}{M^{2}+\frac{n^{2} \pi^{2}}{d^{2}}}+\left\{\frac{1+k s_{1}}{s_{1}-s_{2}} \cdot \frac{s_{1} T^{2}}{s_{1}^{2} T^{2}+\pi^{2}}-\frac{1+k s_{2}}{s_{1}-s_{2}} \cdot \frac{s_{2} T^{2}}{s_{2}^{2} T^{2}+\pi^{2}}\right\}\right. \\
& -2\left(\frac{1+k s_{1}}{s_{1}-s_{2}}\right) \cdot \frac{s_{1} T^{2} \pi^{2}}{s_{1}^{2} T^{2}+\pi^{2}} \cdot \frac{1}{4 s_{1} T} \sum_{m=1}^{\infty} \frac{2\left(\frac{s_{1} T}{2}\right)}{\left(\frac{s_{1} T}{2}\right)^{2}+m^{2} \pi^{2}} \\
& \left.+2\left(\frac{1+k s_{2}}{s_{1}-s_{2}}\right) \cdot \frac{s_{2} T^{2} \pi^{2}}{s_{2}^{2} T^{2}+\pi^{2}} \cdot \frac{1}{4 s_{2} T} \sum_{m=1}^{\infty} \frac{2\left(\frac{s_{2} T}{2}\right)}{\left(\frac{s_{2} T}{2}\right)^{2}+m^{2} \pi^{2}}\right] \\
& =\frac{4 n(-1)^{n}}{d^{2}}\left[\frac{1}{M^{2}+\frac{n^{2} \pi^{2}}{d^{2}}}+\left\{\frac{1+k s_{1}}{s_{1}-s_{2}} \cdot \frac{s_{1} T^{2}}{s_{1}^{2} T^{2}+\pi^{2}}-\frac{1+k s_{2}}{s_{1}-s_{2}} \cdot \frac{s_{2} T^{2}}{s_{2}^{2} T^{2}+\pi^{2}}\right\}\right. \\
& -\frac{1}{2 s_{1} T} \cdot\left(\frac{1+k s_{1}}{s_{1}-s_{2}}\right) \cdot \frac{s_{1} T^{2} \pi^{2}}{s_{1}^{2} T^{2}+\pi^{2}}\left\{\operatorname{coth}\left(\frac{s_{1} T}{2}\right)-\frac{2}{s_{1} T}\right\}
\end{aligned}
$$




$$
\begin{aligned}
& \left.+\frac{1}{2 s_{2} T}\left(\frac{1+k s_{2}}{s_{1}-s_{2}}\right) \cdot \frac{s_{2} T^{2} \pi^{2}}{s_{2}^{2} T^{2}+\pi^{2}}\left\{\operatorname{coth}\left(\frac{s_{2} T}{2}\right)-\frac{2}{s_{2} T}\right\}\right] \\
& =\frac{4 n(-1)^{n}}{d^{2}}\left[\frac{1}{M^{2}+\frac{n^{2} \pi^{2}}{d^{2}}}+\left\{\frac{1+k s_{1}}{s_{1}-s_{2}} \cdot \frac{s_{1} T^{2}}{s_{1}^{2} T^{2}+\pi^{2}}-\frac{1+k s_{2}}{s_{1}-s_{2}} \cdot \frac{s_{2} T^{2}}{s_{2}^{2} T^{2}+\pi^{2}}\right\}\right. \\
& -\frac{1}{2} \cdot\left(\frac{1+k s_{1}}{s_{1}-s_{2}}\right) \cdot \frac{\pi^{2} T}{s_{1}^{2} T^{2}+\pi^{2}} \operatorname{coth}\left(\frac{s_{1} T}{2}\right) \\
& +\frac{1}{s_{1}} \cdot\left(\frac{1+k s_{1}}{s_{1}-s_{2}}\right) \cdot \frac{\pi^{2}}{s_{1}^{2} T^{2}+\pi^{2}} \\
& \left.+\frac{1}{2} \cdot\left(\frac{1+k s_{2}}{s_{1}-s_{2}}\right) \cdot \frac{\pi^{2} T}{s_{2}^{2} T^{2}+\pi^{2}} \operatorname{coth}\left(\frac{s_{2} T}{2}\right)-\frac{1}{s_{2}}\left(\frac{1+k s_{2}}{s_{1}-s_{2}}\right) \cdot \frac{\pi^{2}}{s_{2}^{2} T^{2}+\pi^{2}}\right] \\
& =\frac{4 n(-1)^{n}}{d^{2}}\left[\frac{1}{s_{1} s_{2}}+\left\{\frac{1+k s_{1}}{s_{1}\left(s_{1}-s_{2}\right)}-\frac{1+k s_{2}}{s_{2}\left(s_{1}-s_{2}\right)}\right\}\right. \\
& -\frac{1}{2}\left(\frac{1+k s_{1}}{s_{1}-s_{2}}\right) \cdot \frac{\pi^{2} T}{s_{1}^{2} T^{2}+\pi^{2}} \operatorname{coth}\left(\frac{s_{1} T}{2}\right) \\
& \left.+\frac{1}{2}\left(\frac{1+k s_{2}}{s_{1}-s_{2}}\right) \cdot \frac{\pi^{2} T}{s_{2}^{2} T^{2}+\pi^{2}} \operatorname{coth}\left(\frac{s_{2} T}{2}\right)\right] \\
& =\frac{4 n(-1)^{n}}{d^{2}}\left[\frac{1}{s_{1} s_{2}}-\frac{1}{s_{1} s_{2}}-\frac{1}{2}\left(\frac{1+k s_{1}}{s_{1}-s_{2}}\right) \cdot \frac{\pi^{2} T}{s_{1}^{2} T^{2}+\pi^{2}} \operatorname{coth}\left(\frac{s_{1} T}{2}\right)\right. \\
& \left.+\frac{1}{2}\left(\frac{1+k s_{2}}{s_{1}-s_{2}}\right) \cdot \frac{\pi^{2} T}{s_{2}^{2} T^{2}+\pi^{2}} \operatorname{coth}\left(\frac{s_{2} T}{2}\right)\right] \\
& =\frac{2 n(-1)^{n+1}}{d^{2}}\left[\left(\frac{1+k s_{1}}{s_{1}-s_{2}}\right) \cdot \frac{\pi^{2} T}{s_{1}^{2} T^{2}+\pi^{2}} \operatorname{coth}\left(\frac{s_{1} T}{2}\right)\right. \\
& \left.-\left(\frac{1+k s_{2}}{s_{1}-s_{2}}\right) \cdot \frac{\pi^{2} T}{s_{2}^{2} T^{2}+\pi^{2}} \operatorname{coth}\left(\frac{s_{2} T}{2}\right)\right]
\end{aligned}
$$

Therefore,

$$
\begin{aligned}
& S_{t}=\sum_{n=1}^{\infty}\left\{W_{n}^{\prime}(0) \frac{e^{m_{1} t}-e^{m_{2} t}}{m_{1}-m_{2}}+W_{n}(0) \frac{m_{1} e^{m_{2} t}-m_{2} e^{m_{1} t}}{m_{1}-m_{2}}\right\} \sin \frac{n \pi y}{d} \\
& S_{t}=\sum_{n=1}^{\infty}\left\{W_{n}^{\prime}(0) \frac{e^{s_{1} t}-e^{s_{2} t}}{s_{1}-s_{2}}+W_{n}(0) \frac{s_{1} e^{s_{2} t}-s_{2} e^{s_{1} t}}{s_{1}-s_{2}}\right\} \sin \frac{n \pi y}{d}
\end{aligned}
$$




$$
\begin{aligned}
& =\sum_{n=1}^{\infty}\left[\left\{\frac{W_{n}^{\prime}(0)-s_{2} W_{n}(0)}{s_{1}-s_{2}}\right\} e^{s_{1} t}+\left\{\frac{s_{1} W_{n}(0)-W_{n}^{\prime}(0)}{s_{1}-s_{2}}\right\} e^{s_{2} t}\right] \sin \frac{n \pi y}{d} \\
& =\sum_{n=1}^{\infty}\left[\frac { 2 n ( - 1 ) ^ { n + 1 } } { d ^ { 2 } } \left\{\frac{1+k s_{1}}{s_{1}-s_{2}} \cdot \frac{\pi^{2} T}{s_{1}^{2} T^{2}+\pi^{2}} \cdot \operatorname{coth}\left(\frac{s_{1} T}{2}\right) \cdot e^{s_{1} t}\right.\right. \\
& \left.\left.-\frac{1+k s_{2}}{s_{1}-s_{2}} \cdot \frac{\pi^{2} T}{s_{2}^{2} T^{2}+\pi^{2}} \cdot \operatorname{coth}\left(\frac{s_{2} T}{2}\right) \cdot e^{s_{2} t}\right\}\right] \sin \frac{n \pi y}{d} \\
& =\sum_{n=1}^{\infty}\left[\frac { 2 n ( - 1 ) ^ { n + 1 } } { d ^ { 2 } } \left\{\pi^{2} T \frac{1+k s_{1}}{s_{1}-s_{2}} \operatorname{coth}\left(\frac{s_{1} T}{2}\right) \cdot \frac{e^{s_{1} t}}{s_{1}^{2} T^{2}+\pi^{2}}\right.\right. \\
& \left.\left.-\pi^{2} T \frac{1+k s_{2}}{s_{1}-s_{2}} \operatorname{coth}\left(\frac{s_{2} T}{2}\right) \cdot \frac{e^{s_{2} t}}{s_{2}^{2} T^{2}+\pi^{2}}\right\}\right] \sin \frac{n \pi y}{d} \\
& =\sum_{n=1}^{\infty}\left[\frac { 2 n ( - 1 ) ^ { n + 1 } } { d ^ { 2 } } \left\{\frac{\pi^{2}}{T} \frac{\operatorname{coth}\left(\frac{s_{1} T}{2}\right)}{a_{1}+\frac{b_{1}}{\left(1+k s_{1}\right)^{2}}} \cdot \frac{e^{s_{1} t}}{s_{1}^{2}+\frac{\pi^{2}}{T^{2}}}\right.\right. \\
& \left.\left.+\frac{\pi^{2}}{T} \frac{\operatorname{coth}\left(\frac{s_{2} T}{2}\right)}{a_{1}+\frac{b_{1}}{\left(1+k s_{2}\right)^{2}}} \cdot \frac{e^{s_{2} t}}{s_{2}^{2}+\frac{\pi^{2}}{T^{2}}}\right\}\right] \sin \frac{n \pi y}{d} \\
& =-\frac{2 \pi^{2}}{T d^{2}} \sum_{n=1}^{\infty}\left[n ( - 1 ) ^ { n } \left\{\frac{\operatorname{coth}\left(\frac{s_{1} T}{2}\right)}{a_{1}+\frac{b_{1}}{\left(1+k s_{1}\right)^{2}}} \cdot \frac{e^{s_{1} t}}{s_{1}^{2}+\frac{\pi^{2}}{T^{2}}}\right.\right. \\
& \left.\left.+\frac{\pi^{2}}{T} \frac{\operatorname{coth}\left(\frac{s_{2} T}{2}\right)}{a_{1}+\frac{b_{1}}{\left(1+k s_{2}\right)^{2}}} \cdot \frac{e^{s_{2} t}}{s_{2}^{2}+\frac{\pi^{2}}{T^{2}}}\right\}\right] \sin \frac{n \pi y}{d} \\
& =-\frac{2 \pi^{2}}{T d^{2}} \sum_{n=1}^{\infty} n(-1)^{n} \cdot D \cdot \sin \frac{n \pi y}{d}
\end{aligned}
$$

where

$$
\begin{gathered}
a_{1}=\frac{1}{k}, \quad b_{1}=\left(M^{2}-\frac{1}{k}\right)(1-k) \quad \text { and } \quad D=D_{1}+D_{2}, \\
D_{j}=\frac{e^{s_{j} t} \operatorname{coth}\left(\frac{s_{j} T}{2}\right)}{\left(s_{j}^{2}+\frac{\pi^{2}}{T^{2}}\right)\left(a_{1}+\frac{b_{1}}{\left(1+k s_{j}\right)^{2}}\right)}, \quad j=1,2 .
\end{gathered}
$$

which coincides with the transient part of the equation (4.25) when $s_{1}=m_{1}$, $s_{2}=m_{2}$. 
Thus the two solutions (4.13) and (4.25) are the same for all values of $y$, $k, M$ and $t$. This implies that the method of Fourier analysis which is simpler than that of Laplace transforms can be used successfully to solve similar types of problems.

\section{REFERENCES}

[1] A. Chakraborty and J. Ray, Unsteady magnetohydrodynamic Couette flow between two plates when one of the plates is subjected to random pulses. J. Phys. Soc. Jpn., 48 (1980), 1361.

[2] M.N. Makar, Magnetohydrodynamic flow between two plates when one of the plates is subjected to tooth pulses. Acta Phys. Pol., A71 (1987), 995.

[3] A.R. Bestman and F.I. Njoku, On hydromagnetic channel flow induced by tooth pulses. Preprint IC/88/10, Miramare-Trieste, (1988).

[4] A.K. Ghosh and L. Debnath, On hydromagnetic pulsatile flow of a two-phase fluid. ZAMM, 76(2) (1996), 121.

[5] N. Datta, D.C. Dalal and S.K. Misra, Unsteady heat transfer to pulsatile flow of a dusty viscous incompressible fluid in a channel. Int. J. Heat Mass Transfer, 36(7) (1993), 1783.

[6] N. Datta and D.C. Dalal, Pulsatile flow and heat transfer of a dusty fluid through an infinitely long annular pipe. Int. Multiphase Flow, 21(3) (1995), 515.

[7] T. Hayat, A.M. Siddiqui and S. Asghar, Some simple flows of an Oldroyd-B fluid. Int. J. Engg. Sci., 39 (2001), 135.

[8] S. Asghar, S. Parveen, S. Hanif, A.M. Siddiqui and T. Hayat, Hall effects on the unsteady hydromagnetic flows of an Oldroyd-B fluid. Int. J. Engg. Sci., 41 (2003), 609.

[9] T. Hayat, S. Nadeem and S. Asghar, Hydromagnetic Couette flow of an Oldroyd-B fluid in a rotating system. Int. J. Engg. Sci., 42 (2004), 65.

[10] E.F. El-Shehawey, E.M.E. Elbarbary, N.A.S. Afifi and M. Elshahed, MHD flow of an ElasticoViscous fluid under periodic body acceleration. Int. J. Math. and Math. Sci., 23(11) (2000), 795.

[11] V.M. Soundalgekar, On the flow of an electrically conducting, incompressible fluid near an accelerated plate in the presence of a parallel plate, under transverse magnetic field. Proc. Indian Acad. Sci., A65 (1967), 179.

[12] H.S. Carslaw and J.C. Jaeger, Operational Methods in Applied Mathematics. Dover Publication, Inc., New York (1963).

[13] A.K. Ghosh and P. Sana, On hydromagnetic channel flow of an Oldroyd-B fluid induced by tooth pulses. Magnetohydrodynamics, 44(3) (2008), 325-340.

[14] D.E. Winget, The status of white dwarf asteroseismology and a glimpse of the road ahead. Baltic Astronomy, 4 (1995), 129-136. 
[15] S.V. Koshevaya, V.V. Grimalsky, J. Siqueiros-Alatorre, R. Perez-Enriquez and A.N. Kotsarenko, Acoustic and Acousto-Gravity Wave Pulses Caused by Sources of Seismic Origin. Physica Scripta, 70 (2004), 72-78.

[16] J.J. Condon and R.R. Schmidt, Planetary tides and sunspot cycles. Solar Physics, 42 (1975), 529-532.

[17] K.S. Mekheimer and Y. Abdelmaboud, Peristaltic transport of a particle-fluid suspension through a uniform and non-uniform annulus. Applied Bionics and Biomechanics, 5(2), (June 2008), 47-57.

[18] T.H. Lin, M. Belser and Yonhua Tzeng, Pulsed microwave plasma etching of polymers in Oxygen and Nitrogen for microelectronic applications. IEEE, Transaction on Plasma Science, 16(6), (Dec. 1998), 631-637.

[19] Kang-Ping Lin and W.H. Chang, Adaptive noise reduction for pulmonary artery blood pressure. Engineering in medicine and Biology Society, Proceedings of the Annual International Conference of the IEEE, Vol. I, (1988), 82-83.

[20] Richard Swan, Generating Musical Sounds With Wavelab. http://www.articlesbase.com/ software-articles/ generating-musical-sounds-with-wavelab-441813.html $</ \mathrm{a}></ \mathrm{p}>$. 